Activités

11-2 | Octobre 2014

Varia

\title{
Vidéo formation et transformations de l'activité professionnelle
}

Video training and processes that transform professional activity

\section{Serge Leblanc}

\section{(2) OpenEdition}

\section{Journals}

Édition électronique

URL : http://journals.openedition.org/activites/968

DOI : 10.4000/activites.968

ISSN : 1765-2723

\section{Éditeur}

ARPACT - Association Recherches et Pratiques sur les ACTivités

Référence électronique

Serge Leblanc, "Vidéo formation et transformations de l'activité professionnelle », Activités [En ligne], 11-2 | Octobre 2014, mis en ligne le 15 octobre 2014, consulté le 30 avril 2019. URL : http:// journals.openedition.org/activites/968; DOI : 10.4000/activites.968

\section{(9) $\odot \Theta \Theta$}

Activités est mis à disposition selon les termes de la licence Creative Commons Attribution - Pas d'Utilisation Commerciale - Pas de Modification 4.0 International. 


\title{
Vidéo formation et transformations de l'activité professionnelle
}

\author{
Serge Leblanc \\ Faculté d'Éducation de l'université de Montpellier 2, LIRDEF, 2, place Marcel-Godechot, BP 4152, \\ F-34092 MONTPELLIER Cedex 5 - serge.leblanc@fde.univ-montp2.fr
}

\begin{abstract}
Video training and processes that transform professional activity. This paper presents the main results of a longitudinal study that reveals how a novice teacher can gain work experience by proxy and by anticipation via a platform of online video training focused on analysis of the real work of teachers. To track changes in activity potentially induced by several free navigations in the presence and with the help of a researcher-interlocutor, the "course of life" of a teacher, new to the profession, in relation to the use of this platform (Theureau, 2004), was rebuilt from an ad hoc methodology. Analysis was conducted by linking consulted video resources, concurrent verbalizations during the sessions and, one month later, feedback from the experience. The results show a) local analysis of the development of a self-prescription related to the teacher's professional background, b) an overview of the reconstruction of the main professional tensions experienced in the first six months of class and revealed during navigation on the platform, and c) the effects produced on the teacher's class activity through monitoring of some of these self-prescriptions. Based on the concepts of enaction (Varela, 1989) and individuation (Simondon, 2005), we discuss these results and we propose a vision of learning-development and of integration processes within a group.
\end{abstract}

\section{KEY WORDS}

teacher training, activity analysis, course of life, mimetic experience, allo-confrontation

\section{1.- Introduction}

La spécificité de la formation professionnelle est de chercher à articuler une double transformation de l'activité : celle en situation de formation et celle en situation professionnelle. L'activité débutante se transforme en général de manière importante dans les premiers mois et les premières années d'exercice professionnel. Elle est marquée par une succession d'états passagers ou de compromis provisoires caractérisés par une apparente stabilité. Or si la transformation en cours n'est pas forcément visible, elle peut s'opérer imperceptiblement, en silence sans se faire remarquer pour, quelques mois après, apparaître de manière spectaculaire. L'utilisation de support vidéo en formation, sous certaines conditions éthiques, méthodologiques, de scénarisations pédagogiques et d'accompagnement, peut contribuer à ce processus de développement professionnel à la fois en orientant vers tels ou tels types de transformations, en les accélérant et en rendant certains aspects de ces modifications en cours visibles.

Une récente revue de la littérature scientifique internationale sur l'utilisation de la vidéo dans la formation des enseignants (Gaudin, 2014 ; Gaudin, \& Chaliès, 2012) montre qu'elle se 
développe actuellement de manière accrue aussi bien pour contribuer au développement de leurs compétences disciplinaires spécifiques (par exemple pour l'enseignement de l'éducation physique: Prusak, Graham, \& Graser, 2010 ; pour l'enseignement des mathématiques: Santagata, 2009) que de compétences professionnelles transversales (Brunvand, \& Fishman, 2006 ; Ria, \& Leblanc, 2012 ; Seidel, Blomberg, \& Renkl, 2013). Que ce soit au sein de la formation initiale des enseignants où elle est très utilisée (Calandra, \& Puvirajah, 2011), mais aussi en formation continue (van Es, \& Sherin, 2008), de nombreuses études pointent des effets bénéfiques notamment en termes a) d'attractivité chez les enseignants qui vivent à cette occasion une expérience authentique renforçant leur intérêt pour le métier (Sherin, 2004), b) de réflexivité conduisant à des commentaires spécifiques plus en lien avec les pratiques réelles (comparativement à une réflexion écrite de type mémoire), et c) d'évolution de leurs préoccupations centrées d'abord sur la gestion de classe et sur l'usage d'eux-mêmes puis se focalisant avec un peu plus d'expérience sur l'enseignement et sur les élèves (Rosaen, Lundeberg, Cooper, Fritzen, \& Terpstra, 2008).

La construction des dispositifs de formation utilisant des ressources vidéo s'opère principalement selon deux approches: soit une approche prescriptive qui vise à faire construire des connaissances pédagogiques en visionnant les «bonnes pratiques» d'enseignement pour répondre à la question «que faire» dans la classe (Brouwer, 2011), soit une approche développementale qui vise à stimuler une réflexion professionnelle en confrontant les enseignants à de multiples pratiques de classe (Sherin, 2004). En étant structurée sur la distinction voire l'opposition «expert-novice », la première approche laisse totalement dans l'ombre le processus de constitution de cette expertise. En ne s'appuyant pas forcément sur un ancrage théorique particulier pour élaborer un objectif de visionnage vidéo, la deuxième approche prend le risque de développer des discussions professionnelles anecdotiques et/ou des analyses superficielles. Pour tenter de limiter ces biais, un certain nombre de travaux francophones sur la vidéo formation ont renouvelé ces approches en redéfinissant les objectifs de visionnage vidéo à partir des cadres d'analyse du travail (Faïta, 2007 ; Flandin, 2014 ; Flandin, \& Ria, 2013 ; Gaudin, 2014 ; Gaudin, \& Chaliès, 2012 ; Leblanc, \& Veyrunes, 2012 ; Moussay, 2013 ; Ria, \& Leblanc, 2011).

Certaines de ces études récentes sur l'utilisation de ressources vidéo d'enseignants débutants dans le métier ont pointé d'autres effets sur l'activité des formés en formation initiale (Leblanc, 2012, Leblanc, \& Sève, 2012; Lussi-Borer, \& Muller, sous presse; Ria, \& Leblanc, 2012) : a) un effet de réassurance et de déculpabilisation des enseignants débutants grâce à une prise de conscience des passages plus ou moins incontournables de l'appropriation du métier, b) un effet de rappel spontané d'expériences vécues favorisé par les situations de classe vidéoscopées qui possèdent un «air de famille » avec leurs propres pratiques professionnelles, c) un effet de comparaison entre les expériences présentées à travers les vidéos et les expériences vécues par les utilisateurs leur permettant d'évaluer leur propre pratique en se voyant à travers autrui, et d) un effet de projection les conduisant à anticiper des scénarios non rencontrés jusque-là et à envisager d'autres possibles à tester concrètement dans leurs propres classes en s'appuyant sur l'expérience acquise par d'autres pairs.

Notre contribution se situe dans le prolongement de ces travaux. Des dispositifs de vidéo formation conçus à partir de films du travail réel ayant une proximité avec le vécu des formés et minimalement montés pourraient contribuer, sous certaines conditions, à stimuler, anticiper et accompagner des apprentissages professionnels. Ces environnements solliciteraient l'articulation de processus mimétiques, réflexifs et projectifs sur la base d'une activité de comparaison entre ce que les enseignants débutants en formation perçoivent d'une situation filmée-commentée et ce qu'ils font dans leur classe. Mais cette comparaison resterait le plus souvent implicite en formation, peu explorée et dans le domaine des pensées privées des enseignants. Les chercheurs seraient, quant à eux, en difficulté pour déconstruire et reconstruire un processus complexe de mises en relation d'expériences vécues par les enseignants dans des espaces différents (en formation et au travail) et sur des temporalités 
longues (un semestre, une ou plusieurs années). De plus, ce processus de «transformations silencieuses » (Jullien, 2009) de leurs mondes d'expériences ${ }^{1}$ serait peu conscientisé par les acteurs et de ce fait encore plus délicat à appréhender.

À partir d'une étude longitudinale ${ }^{2}$, nous chercherons à montrer comment de l'expérience professionnelle peut s'acquérir par procuration et anticipation via ces environnements de vidéo formation centrés sur l'analyse du travail réel des enseignants et quels types d'apprentissages sont construits à travers les expériences que cela génère ?

\section{2.- Cadre conceptuel}

Une des évolutions majeures des pratiques de formation notamment aux métiers de la relation (enseignement, soins, éducation) a été l'émergence de l'analyse de pratique dans les formations (Éducation Permanente, 2005) fondée à partir des concepts de "praticien réflexif » (Schön, 1994) et/ou d' "approche clinique »(Cifali Bega, 2009). Ces dispositifs se sont construits probablement en écho à une double évolution, premièrement, praxéologique avec la centration de la formation professionnelle sur les compétences à acquérir, notamment dans le cadre de l'alternance pédagogique (Perrenoud, 2001), et deuxièmement, épistémologique avec la montée en puissance du paradigme de l'action et de la cognition située (Lave, 1988, Suchman, 1987), des théories de l'activité (Amalberti, de Montmollin, \& Theureau, 1991 ; Clot, 1999; Leplat, 1997) et de recherches en éducation et en formation menées à partir de ces concepts (Barbier, \& Durand, 2003 ; Leblanc, Ria, \& Veyrunes, 2012 ; Linard, 2002). Cette approche dite «ascendante 》 (Méard, 2004) a donné naissance tout d'abord à de nombreux dispositifs d'analyse de pratiques dans la formation des enseignants fondés davantage sur des discours a posteriori ou sur des écrits à propos de la pratique que sur des supports vidéo ${ }^{3}$.

$\mathrm{Si}$ ces dispositifs permettent d'analyser des situations singulières et de répondre individuellement aux préoccupations immédiates des stagiaires, trois difficultés subsistent. Premièrement, un discours sur la pratique se réduit aux catégories usuelles du langage technique du domaine professionnel et aux catégories d'analyse de la tâche basées sur un «point de vue extrinsèque ». Il ne restitue donc pas ce qui est réellement vécu et réalisé dans une situation. Pour dépasser ce décalage entre ce que l'on dit et ce que l'on fait, des techniques de «verbalisation consécutive assistée » (Leplat, \& Hoc, 1981) ont été progressivement mises au point pour expliciter l'action notamment en développant des techniques de guidage de l'entretien (Vermersch, 2006) et/ou différents modes de confrontation à des traces de l'activité (Mollo, \& Falzon, 2004 ; Rix-Lièvre, 2010 ; Theureau, 2010). Deuxièmement, en étant enfermés dans une approche idiosyncrasique (Durand, de Saint Georges, \& Meuwly-Bonte, 2006), ces dispositifs d'analyse de pratiques répondent de manière insatisfaisante aux difficultés récurrentes auxquelles les enseignants vont être confrontés dans leurs premières années professionnelles dans la mesure où il est

1 Le monde d'expérience correspond au « monde propre » (Merleau-Ponty, 1942) à partir duquel un enseignant agit ; il ne peut être défini indépendamment de la façon dont il perçoit la situation, de ce qu'il cherche à faire, de ce qu'il ressent et de ce qu'il mobilise comme connaissances en relation avec ce qu'il fait. Ainsi, le monde d'un enseignant débutant dans le métier ne coïncide que partiellement avec celui d'un enseignant expérimenté, le premier vit les situations en permanence comme nouvelles et donc comme fortement anxiogènes alors que le deuxième reconnaît régulièrement des situations déjà vécues à partir d'indices typiques.

2 Les études longitudinales centrées sur les transformations de l'activité d'enseignants en formation en lien avec le contexte professionnel sont peu nombreuses (e.g. Serres, 2006).

3 Cette analyse est extraite de la Conférence de consensus intitulée «L'analyse des pratiques dans la formation des enseignants » qui a eu lieu à l'IUFM de Créteil le 25 janvier 2006 et qui a fait l'objet d'un rapport. http://www.creteil.iufm.fr/fileadmin/documents/siteFFO/Service/Productions/2006_Conf_cons.pdf (Consulté le 24 février 2011). 
nécessaire d'attendre d'avoir vécu un problème professionnel pour pouvoir l'analyser et envisager des solutions non encore explorées ${ }^{4}$. Troisièmement, en ayant recours à la narration ou à l'écriture, ils restituent le travail comme un objet déjà structuré par le langage et ainsi déjà «trop » normé (Quéré, 1993).

L'approche de la conception de dispositif de formation «orientée-activité » (Leblanc, Ria, Dieumegard, Serres, \& Durand, 2008) dans laquelle s'inscrit ce travail se veut une alternative en se focalisant sur le traitement de thématiques critiques pour des entrants dans le métier conçus à partir de matériels vidéo de recherches sur les enseignants débutants (e.g. Félix, \& Saujat, 2008 ; Ria, 2009 ; Veyrunes, 2011). Le principe est d'aider les enseignants à anticiper des transformations de leur activité dans leurs premiers mois et années d'enseignement en les confrontant aux meilleurs exemplaires qui illustrent telles ou telles façons de faire ou difficultés typiques des débutants (Leblanc, \& Veyrunes, 2012) et en construisant des scénarios de vidéo formation au sein d'un processus progressif de professionnalisation (Leblanc, 2014a). L'approche s'inspire des outils de formation et des situations de simulation développés en didactique professionnelle et en ergonomie sur la base de l'analyse des conceptualisations dans l'activité réelle qui permettent de confronter les acteurs à une approche d'apprentissage-développement de situations favorisant cette anticipation (Mayen, 1999 ; Ouellet, 2013 ; Pastré, 1995, 2005). Ils favorisent la construction d'une expérience «suffisamment» analogue à celle d'une situation réelle (tout au moins dans ses dimensions cognitives) tout en manipulant des paramètres de la situation supportant l'apprentissage: suppression du risque, ajustement de la complexité en termes de temporalité, d'étendue de l'environnement, de degré de difficulté des problèmes rencontrés (Caens-Martin, Specogna, Delépine, \& Girerd, 2004). L'approche s'inspire également du courant de la clinique de l'activité (Faïta, 2007) qui utilise les enregistrements vidéo dans le cadre d'une démarche de mise en développement des enseignants. La vidéo sert d'outil d'investigation pour les sujets, confrontés à leurs propres réussites ou échecs professionnels et offre des moyens inédits d'interpréter des situations concrètes les impliquant et de les transformer par le «redoublement de l'expérience vécue» dans le cadre dialogique de l'auto-confrontation. L'extension du travail d'analyse à un collectif d'enseignants plus ou moins expérimentés contribue au développement de controverses professionnelles révélatrices d'une dynamique du métier. Notre approche «orientée-activité » de l'alternance fait de l'expérience en situation professionnelle ou du travail enseignant analysé et modélisé par les travaux de recherche une ressource pour la formation et l'accompagnement du développement professionnel (Bertone, 2011 ; Durand, Ria, \& Veyrunes, 2010 ; Étienne, Altet, Lessard, Paquay, \& Perrenoud, 2009 ; Étienne, \& Bucheton, 2009 ; Faïta, \& Saujat, 2010 ; Leblanc, 2007 ; Leblanc et al., 2008 ; Leblanc \& Ria, 2014 ; Mayen, 2009).

Pour appréhender, rendre compte et comprendre ces transformations de l'activité en lien avec les caractéristiques des environnements de vidéo formation, notre cadre est fondé sur quatre hypothèses théoriques : a) l' "expérience fictionnelle », c'est-à-dire le jeu entre le réel du travail et l'environnement d'apprentissage/développement (Durand, 2009), constitue un vecteur de transformation de l'expérience qu'il s'agit de comprendre afin de l'exploiter au mieux dans les situations de formation; b) la vidéo est un objet temporel qui possède la particularité d'avoir une structure d'écoulement semblable à celle de notre conscience (Stiegler, 2010). La confrontation à des films de classe et/ou à des entretiens des acteurs à propos de ces films suscite de manière immédiate et spontanée des processus de synchronisation, de création d'attentes et d'anticipation ; c) la navigation dans la plateforme de vidéo formation définit et délimite l'espace des expériences mimétiques possibles pour les utilisateurs-acteurs. L'espace mimétique est créé par l'interaction entre le visionnement

4 Cette critique est particulièrement valable en formation initiale pour un groupe d'acteurs qui débutent professionnellement. Elle ne l'est pas avec un groupe d'acteurs expérimentés qui profitent assez facilement de ces analyses et échanges d'expériences. 
d'extraits filmiques présentant une situation de classe, le vécu professionnel de l'enseignant impliqué dans la vidéo et/ou des commentaires de pairs avec le questionnement du chercheur-interlocuteur qui amène l'utilisateur-acteur à expliciter ses pensées et sentiments à chaque moment significatif pour lui de cette navigation. Cette immersion est à la fois spatiale en plongeant le spectateur dans l'ambiance d'une classe particulière et temporelle, dans la mesure où l'utilisateur découvre pas à pas l'évolution de la situation. Citton parle de «transe de l'immersion » qui "nous force à bouger, à agir et non seulement à observer » (2012, p. 112) ; d) la création de réseaux de relation entre des vécus "par procuration», « rejoués », «imaginés » est favorisée par l'environnement de vidéo formation et l'adressage à autrui du discours privé des utilisateurs (Leblanc, \& Sève, 2012). Cette activité de mise en lien permet de construire de nouvelles structures d'anticipations (en termes de reconnaissance de situations et d'actions à mobiliser) et des connaissances situées potentiellement remobilisables en situation de classe.

Pour pister les transformations de l'activité en formation potentiellement induites par l'utilisation de la plateforme de vidéo formation Néopass@ction ${ }^{5}$ en présence et avec l'aide d'un chercheur-interlocuteur, nous reconstruisons le cours de vie de l'enseignant relatif à l'utilisation de la plateforme en lien avec son contexte professionnel. L'objet théorique du «cours de vie relatif à une pratique» (Theureau, 2006) constitue une extension du cours d'expérience à des périodes d'activités discontinues s'étendant sur des empans temporels plus larges et est particulièrement pertinent pour traiter des activités à long terme qui parcourent la vie d'un acteur au milieu d'autres activités. Par hypothèse, le cours d'expérience restitue le flux des préoccupations, émotions, actions, focalisations, interprétations, connaissances mobilisées et construites... d'un acteur au cours d'une période d'activité (Sève, \& Saury, 2010). La reconstruction de ses différents cours d'expérience permet de pister notamment les préoccupations de l'enseignant et l'évolution de celles-ci en fonction de ce qui fait signe dans les vidéos consultées et/ou des expériences rappelées (représentamen ${ }^{6}$ ). Il s'agit également de repérer les unités de cours d'expérience de type «imagination», et «auto-réflexion» (Theureau, 2006) afin de mieux comprendre la manière dont l'expérience vécue via l'environnement de vidéo formation et celle vécue en classe peuvent potentiellement se nourrir mutuellement en permettant de construire des savoirs situés relatifs aux situations de classe analysées et des savoirs sur soi-même relatifs à la manière de se développer professionnellement. L'imagination, que l'on peut définir comme la faculté de se représenter un objet même en son absence, s'exprime sous différentes formes, à travers des unités de cours d'expérience : a) soit «imaginées-rejouées » sur la base d' «image-souvenir » qui réactive la totalité d'un passé à partir d'un fragment de celui-ci, b) soit «imaginées-recomposées » sur la base d' «image-perçue » de l'activité d'un pair qui fait écho, c) soit «imaginées-créées » sur la base d' «image-esquisse» qui anticipe sur l'activité future. L'auto-réflexion que déploie l'acteur, en cours de navigation sur la plateforme ou lors des bilans relatifs à des expérimentations en classe, gagne à s'ancrer sur l'activité effective de classe et/ou sur une activité «imaginée » en formation comparable ou voisine. En favorisant l'expression de la conscience pré-réflexive de l'activité de l'acteur (en vidéo formation et/ou en classe), on postule que l'activité réflexive qui se développera «aura pour objet quelque chose de proche de cette première activité » (Theureau, 2012, p. 14) et que les pistes de transformation élaborées seront davantage sujettes à traverser les contextes. Quel protocole de recueil de données et quelles modalités d'entretien avons-nous mis en place pour pouvoir documenter précisément ces pistes de transformations projetées et en suivre l'expérimentation en classe ?

5 Plateforme de formation en ligne développée par une équipe de chercheurs (Ria et collaborateurs, 2010) et hébergée au sein de l'Institut Français de l'Éducation de l'École normale supérieure de Lyon.

6 Le représentamen correspond à ce que l'acteur prend en compte, au temps t, dans la situation, pour agir en fonction de sa structure d'attente. Ces éléments peuvent être soit perçus dans l'environnement soit rappelés par des souvenirs qui font écho à l'instant vécu. 


\section{3.- Méthodologie}

Trois sessions de travail avec la plateforme Néopass@ction ont été réalisées par une enseignante-stagiaire de mathématiques nommée Coralie de septembre 2011 à janvier 2012. Cette enseignante s'engage dans la première session pour résoudre une difficulté précise «faire face à certains élèves de $4^{\text {ème }}$ qui adoptent des comportements perturbateurs particulièrement le vendredi après-midi et trouver des gestes professionnels pour essayer de les mettre au travail ». En même temps que Coralie naviguait librement sur la plateforme en présence du chercheur, elle était invitée à produire des «verbalisations simultanées et interruptives» (Theureau, 2004). Au total, trois entretiens de ce type d'une durée d'une heure chacun ont été effectués (Figure 1).

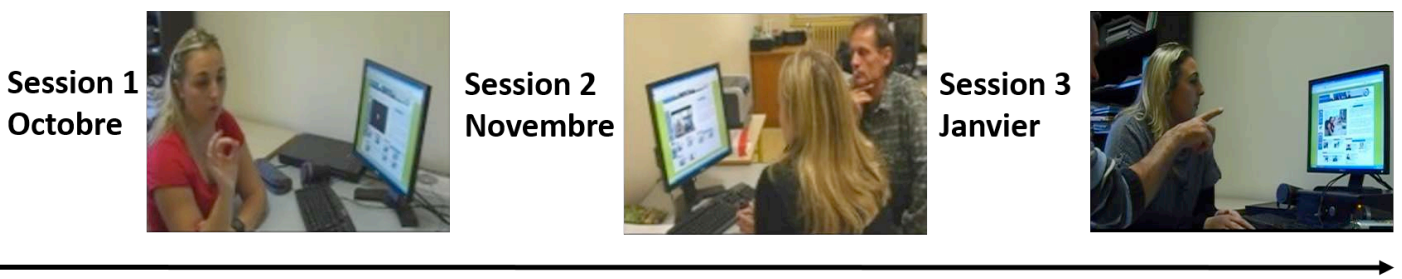

Figure1 : Navigations libres sur la plateforme Néopass@ction couplées à des verbalisations simultanées et interruptives

Figure 1: Free Navigations on the Néopass@ction platform coupled with concurrent and interruptive verbalizations

Les données de verbalisations ont été produites par des questionnements et relances du chercheur-interlocuteur qui a invité Coralie, tout au long de sa navigation, à expliciter son activité dans la plateforme. Ces relances visaient à faciliter a) la description de sa «conscience pré-réflexive » lors de sa navigation, b) l'explicitation du point de vue adopté sur la situation (celui de l'enseignant, des élèves, d'un élève, d'elle-même en tant qu'enseignante...), c) l'engagement et le maintien dans cette situation de formation, et d) l'énoncé de pistes concrètes qu'elle envisageait de tester dans sa classe (auto-prescriptions). Lors de la session de travail suivante (espacée d'environ un mois), l'entretien démarrait sur l'explicitation de ce qui avait été effectivement mis en place en classe à partir des autoprescriptions construites lors de la session précédente. Dans le cas d'une non-mise en œuvre, il était demandé d'en expliquer les raisons.

Les trois navigations commentées sur la plate forme ont fait l'objet d'un enregistrement audio-vidéo (3h), d'une retranscription des verbatim en intégralité et de la réalisation d'un synoptique des vidéos consultées (Annexe 1). L'analyse a consisté a) à construire un protocole à trois volets ${ }^{7}$ permettant d'identifier les transformations projetées à la fin de chaque session de vidéo formation et de «pister » leur réalisation ou non en classe ainsi que leurs effets sur la pratique à travers un retour d'expérience réalisé un mois plus tard, b) à mener une analyse globale sur la base du récit réduit visant à identifier les séquences ${ }^{8}$ organisatrices des pistes de transformations projetées, c) à estimer le caractère de réinvestissement possible des pistes de transformations projetées en repérant les conditions de mise en œuvre envisagées par l'enseignante ainsi que les effets produits lorsqu'elles ont été expérimentées.

7 L'organisation des données en trois volets nous a permis de pister une transformation en cours ou qui émerge en synchronisant les extraits de données en lien direct avec elle (au sein d'une même session et avec la session suivante).

8 Une séquence est une structure significative de rang supérieur qui articule plusieurs unités d'expérience qui regroupent des actions-communications correspondant à un même engagement ou à une même préoccupation. 


\section{1.- Construction du protocole à trois volets}

Cette étape a consisté à construire des protocoles à trois volets pour les unités significatives élémentaires ${ }^{9}$ (USE) qui ont conduit à des projections de transformation des manières d'agir de l'enseignante en situation de classe (Tableau 1). Elles ont été documentées et explicitées dans un premier entretien et ont fait l'objet d'un retour sur expérience dans un deuxième entretien positionné environ un mois plus tard. Le volet 1 correspond aux extraits vidéo significatifs de la plateforme qui ont servi d'ancrage et ont été déclencheur du processus de transformation. Le volet 2 correspond aux commentaires spontanés qu'ils ont générés et aux relances du chercheur effectuées pour documenter les composantes de l'expérience et maintenir cette activité. Le volet 3 correspond à la description des expériences vécues en classe en lien avec ces projections et aux effets repérés de cette transformation de pratique (environ un mois après).

\begin{tabular}{|c|c|c|}
\hline \multicolumn{3}{|c|}{ Transformation 1 : Circuler dans les rangs pendant le rituel de début du cours } \\
\hline Volet 1 & Volet 2 & Volet 3 \\
\hline $\begin{array}{l}\text { Vidéo Enseignante } \\
\text { néotitulaire d'EPS }\end{array}$ & $\begin{array}{l}\text { Verbalisations simultanées et } \\
\text { interruptives }\end{array}$ & $\begin{array}{l}\text { Retour d'expérience un mois plus tard après } \\
\text { expérimentation dans la classe }\end{array}$ \\
\hline $\begin{array}{l}\text { Nous en plus en EPS, on } \\
\text { bouge pas mal, on est } \\
\text { pas mal en plein air où il } \\
\text { faut porter la voix. Donc } \\
\text { c'est vrai que les } \\
\text { moments où on peut ben } \\
\text { retomber, enfin ne pas } \\
\text { parler ou même } \\
\text { économiser la voix, c'est } \\
\text { vrai que c'est assez } \\
\text { intéressant et c'est } \\
\text { reposant aussi de, ben } \\
\text { d'arriver à communiquer } \\
\text { avec eux et de faire } \\
\text { comprendre ce qu'on } \\
\text { veut sans forcément } \\
\text { parler et, et s'agiter dans } \\
\text { tous les sens. }\end{array}$ & $\begin{array}{l}\text { Coralie : Voilà, ça, je ne le fais } \\
\text { pas pour l'instant. Je balaye du } \\
\text { regard, mais effectivement, heu, } \\
\text { comme ils sont debout, des fois } \\
\text { je ne les vois pas tous. Donc, je } \\
\text { pense que je vais heu, heu (...), } \\
\text { je vais bouger dans la classe } \\
\text { pour heu, ben justement montrer } \\
\text { que ben, et plus facilement en } \\
\text { tout cas à ceux qui sont pas en } \\
\text { place, ben, de les faire un petit } \\
\text { peu heu s'activer pour qu'ils se } \\
\text { mettent plus rapidement en } \\
\text { position de travail. }\end{array}$ & $\begin{array}{l}\text { Au niveau de la mise en place, enfin de l'entrée } \\
\text { en classe, euh, ça va mieux parce que les élèves } \\
\text { ils se mettent, assez, un peu plus rapidement en } \\
\text { situation de travail... je le vis positivemen } \\
\text { puisque c'est ce que j'attends en fait. Voilà, on } \\
\text { perd moins de temps. Je ne fais plus l'appel, je } \\
\text { circule dans la classe jusqu'à ce qu'ils aient tous } \\
\text { sorti leurs affaires et puis après, je les fais } \\
\text { asseoir et voilà. Je me suis un petit peu servi de } \\
\text { ce que j'avais vu justement, plus rester devant le } \\
\text { tableau à attendre, mais circuler pour, ben pour } \\
\text { montrer que ben j'attends qu'ils soient, enfin } \\
\text { qu'ils se mettent rapidement en place. Le cours } \\
\text { démarre plus rapidement et les élèves sont plus } \\
\text { calmes plus rapidement, quand les élèves me } \\
\text { voient, ils se taisent automatiquement puisqu'ils } \\
\text { savent que, enfin ils ont un petit peu imprimé } \\
\text { les règles. }\end{array}$ \\
\hline
\end{tabular}

Tableau 1 : Protocole à trois volets pour pister des transformations projetées et expérimentées

Table 1: Three-facetted protocol to track projected and tested transformations

\section{2.- Identification de séquences discontinues sur la base du récit réduit}

Pour identifier toutes les sources d'influence ${ }^{10}$ de ces transformations projetées, nous avons reconstruit l'enchaînement des unités significatives élémentaires (USE) sous forme d'un récit réduit (Annexe 2) et identifié celles qui relevaient d'une même préoccupation à un niveau plus générique. Ces structures significatives de rang supérieur ont permis à la fois de pister les liens construits au fil de la navigation en fonction des vidéos consultées (e.g. propos d'une enseignante d'EPS débutante et image fixe de Romain «adossé au tableau les bras croisés», enseignant en difficulté pour débuter son cours) et également d'estimer l'importance de la préoccupation de l'enseignante à travers le nombre d'occurrences et

9 Les unités significatives élémentaires sont les fractions de l'activité pré-réflexive montrable, racontable et/ou commentable par l'acteur qui émergent de l'articulation dynamique de plusieurs éléments et qui en restituent la signification locale.

10 Les sources d'influence correspondent aux éléments perçus dans l'environnement (e.g. attitude de Romain dans la vidéo) et/ou de l'expérience de l'acteur rappelé (e.g. propos d'une formatrice relatifs à une attitude similaire) qui sont à l'origine de transformations de l'activité ou à l'inverse de l'arrêt de ce processus (e.g. propos de la tutrice qui diminue la force de conviction des pistes construites lors de la navigation). Elles sont toujours évoquées par l'acteur lui-même et reconstruites par le chercheur. 
l'épaisseur du discours restitué notamment au niveau des connaissances mobilisées et construites (Tableau 2).

\begin{tabular}{|c|c|c|}
\hline $\mathrm{N}^{\circ}$ & USE & $\begin{array}{l}\text { Analyse } \\
\text { globale }\end{array}$ \\
\hline 28. & $\begin{array}{l}\text { Consulte la vidéo conseillée par le chercheur (suite à sa demande) d'une } \\
\text { professeure d'EPS qui commente la première vidéo de classe de Romain } \\
\text { (enseignant en difficulté pour gérer le début de son cours) }\end{array}$ & \multirow{8}{*}{$\begin{array}{l}\text { Séquence : } \\
\text { Contrôler la } \\
\text { mise en position } \\
\text { scolaire de tous } \\
\text { les élèves lors } \\
\text { du rituel de } \\
\text { début de } \\
\text { cours en } \\
\text { ajustant sa } \\
\text { propre manière } \\
\text { d'agir }\end{array}$} \\
\hline 29. & $\begin{array}{l}\text { Exprime son accord avec les propos de la collègue sur les conditions à } \\
\text { respecter pour que l'attitude «les bras croisés » soit efficace }\end{array}$ & \\
\hline 30 . & $\begin{array}{l}\text { Rejoue ce qu'elle fait dans sa propre classe en décrivant précisément } \\
\text { certaines attitudes d'élèves }\end{array}$ & \\
\hline 31. & $\begin{array}{l}\text { Imagine une alternative à tester dans sa classe qui intègre des pistes issues } \\
\text { des propos de la collègue d'EPS jugées pertinentes pour elle pour que les } \\
\text { élèves se mettent plus rapidement au travail }\end{array}$ & \\
\hline$\ldots$ & & \\
\hline 36. & $\begin{array}{l}\text { Interpellée par l'attitude de Romain adossée au tableau les bras croisés } \\
\text { (image fixe), arrête sa consultation }\end{array}$ & \\
\hline 37. & Se rappelle qu'une formatrice lui avait fait cette même remarque & \\
\hline 38. & $\begin{array}{l}\text { Prend conscience de l'image que cela peut renvoyer aux élèves et de ses } \\
\text { effets }\end{array}$ & \\
\hline
\end{tabular}

Tableau 2: Analyse globale et construction d'une séquence discontinue

Table 2: : Global analysis and construction of a discontinuous sequence

\section{3.- Réinvestissement envisagé des pistes de transformation construites et conditions}

Ces enquêtes menées par l'enseignante ont débouché régulièrement sur la construction de pistes d'action plus ou moins directement expérimentables dans la classe. Le caractère réalisable ainsi que la force de conviction des pistes construites se révèlent à travers les expressions de réinvestissement utilisées par l'enseignante pour l'exploiter dans ses classes. Ces expressions peuvent être positionnées sur un curseur entre doute et certitude. Elles expriment a) une interrogation sur le contexte d'exploitation «Après, je me demande si c'est possible de faire ça à chaque fois (...) ou si ça doit rester, je sais pas, une fois par semaine par exemple ou heu, ou à des heures difficiles», b) la nécessité d'une adaptation disciplinaire "ça peut être pas mal heu, parce que moi en plus, j'ai un vidéoprojecteur dans ma salle (...), mais je réfléchis si je peux le faire, en maths », c) une tension entre des préoccupations qui sont perçues comme incompatibles ${ }^{11}$ "je vais, je peux prévoir des exercices justement au cas où... justement, au cas où ben il y ait cette sanction-là à donner. Après voilà, moi ce qui me, ce qui m'embête un peu c'est l'aspect collectif, mais », d) un intérêt souligné pour une façon de faire qui lui apparaît tout de suite pertinente et exploitable à son niveau "c'est vrai que là c'est une bonne idée d'envoyer trois élèves au tableau (...) oui, oui, ça peut être pas mal. J'aurais dî le regarder avant», e) une projection dans des temporalités plus ou moins longues dans la mesure où certaines modalités d'intervention nouvelles doivent être suffisamment réfléchies avant d'être testées "Ce que je vais modifier c'est le cartable des 6e, mais je peux le faire de suite, enfin de suite, je ne vais pas attendre après les vacances (...) Et après pour les 4 e, à la rentrée, je vais essayer de..., de faire passer des élèves pour la correction pendant que moi... », f) une expérimentation limitée à une seule classe compte tenu de l'appréhension que suscite cette nouvelle modalité et de la

11 Face à une ambiance de classe perturbée par un groupe important d'élèves, la solution consistant à reprendre la main en imposant à toute la classe des exercices-sanction peut être ressentie de manière injuste par certains élèves. Coralie interroge les limites de cette piste d'intervention car elle perçoit une contradiction possible entre deux préoccupations « reprendre le contrôle de la classe » et «être juste vis à vis des élèves nonperturbateurs ». 
nécessité de la tester avant de la généraliser "Comme c'est inconnu pour l'instant, c'est vrai que c'est ce qui me fait peur un petit peu, mais bon, je veux dire, ce n'est pas... voilà, c'est faisable de toute façon donc (...) une classe sur les quatre pour voir un petit peu, enfin faire la comparaison entre ce que ça apporte ».

\section{4.- Résultats : Cours de vie de Coralie relatif à l'utilisation de la plateforme en lien avec son contexte professionnel}

Cette contribution présente la reconstruction de l'enchaînement des différentes expériences relatives à l'utilisation de la plateforme (trois sessions étalées sur une période de 4 mois) en lien avec son contexte professionnel qui nous a permis de suivre quelques transformations significatives de l'activité en classe de cette enseignante débutante. Pour cela, nous analysons premièrement l'élaboration d'une transformation en lien avec son contexte professionnel via la première navigation dans la plateforme. Deuxièmement, nous présentons une vision synthétique de la reconstruction des principales tensions professionnelles qui se sont révélées lors de ces trois navigations commentées en écho à ses premiers pas professionnels. Nous nous sommes focalisés sur celles qui avaient débouché sur des autotransformations projetées en lien avec son contexte professionnel. Troisièmement, nous montrons quelques effets produits sur l'activité et le développement professionnel à partir du suivi de certaines de ces auto-transformations dans la classe.

Les résultats présentés apportent des éléments de réponse aux questions suivantes: Quels types $^{12}$ sont construits lors de la consultation de la plateforme en lien avec le contexte professionnel de Coralie? Quelles tensions ${ }^{13} \mathrm{chez}$ cette enseignante débutante entre ses expériences de classe et celles de pairs sont actualisées par la consultation des ressources de la plateforme ? À quelle période de l'année apparaissent-elles et à travers la consultation de quels extraits vidéo ? Quels types ont été validés et construits suite à une expérimentation en classe?

\section{1.- Analyse locale d'une transformation projetée de son activité en classe via la première navigation dans la plateforme}

L'analyse du cours d'expérience de Coralie a été construite sur la base du volet 1 et du volet 2. Nous illustrons ici cette analyse en nous focalisant sur une unité d'expérience articulant imagination et autoréflexion qui est constitutive d'une transformation projetée en fin de première session de navigation. Les extraits les plus significatifs pour comprendre la transformation projetée sont présentés ci-dessous (Tableau 3). L'analyse de la transformation est réalisée à partir des éléments du signe hexadique (Tableau 4).

12 Les types sont le résultat d'un processus de typicalisation à travers lequel une personne reconnait certaines expériences singulières comme récurrentes dans des contextes perçus comme similaires. Ils se décrivent comme des « schèmes typiques d'attention, de perception, d'action, de communication, d'interprétation et d'émotions » (Theureau, 2000, p. 185) toujours liés à l'activité en cours.

13 Les tensions sont conscientes ou conscientisables grâce à l'explicitation de la conscience pré-réflexive. Elles correspondent à une opposition ou une compatibilité difficile entre deux préoccupations saillantes au sein de l'activité de l'acteur, à une contradiction entre ce qu'il fait et ce qu'il voudrait faire mais n'arrive pas à faire ou ne s'autorise pas à faire. Elles peuvent être issues de sa propre pratique. Dans cette situation de vidéo formation, elles sont le plus souvent suscitées par la confrontation à des façons de faire différentes de pairs et par la perspective transformative de sa propre activité. Certaines de ses tensions exprimées dans cette situation sont proches de «dilemmes de métier» (Saujat, 2010) dans la mesure où elles sont repérées comme typiques, récurrentes et stables et sont partagées au sein de la communauté enseignante (e.g. nécessité d'un travail d'appropriation des connaissances par les élèves et le risque d'accroître les inégalités entre eux en donnant des devoirs à faire à la maison). 


\begin{tabular}{|c|c|}
\hline $\begin{array}{l}\text { Volet } 1 \text { : Vidéos } \\
\text { significatives }\end{array}$ & Volet 2 : Verbalisations simultanées et interruptives \\
\hline $\begin{array}{l}\text { Vidéo commentaires } \\
\text { de l'enseignante } \\
\text { d'EPS } \\
\text { Située à la } 25^{\text {ème }} \\
\text { minute de la } \\
\text { navigation } \\
\text { commentée }\end{array}$ & $\begin{array}{l}\text { Je suis assez d'accord avec ce qu'elle dit (...) Sur le fait de heu, de rester bon les bras } \\
\text { croisés, heu, sur une même posture, heu. (...) ça marche, mais à condition de soit } \\
\text { balayer là, comme elle dit de balayer la classe du regard ou alors, moi je m'attarde } \\
\text { aussi sur heu, enfin je regarde précisément les élèves qui sont pas en place pour moi. } \\
\text { C'est-à-dire moi je les, quand, quand ils entrent en classe en fait, ils sont debout, ils } \\
\text { restent debout, ils doivent sortir toutes leurs affaires de maths et ensuite, j'attends le } \\
\text { silence pour les faire asseoir. Donc ben là, souvent il y en a, ils sont dans la lune, donc } \\
\text { ils oublient de sortir leurs affaires. Donc, je leur rappelle. Ou il y en a qui s'asseyent, } \\
\text { qui s'assoient avant que je le leur aie demandé, donc, je leur rappelle aussi. Mais par } \\
\text { contre, ce que je pense que je vais heu, je vais faire, c'est effectivement ben passer } \\
\text { dans la classe. }\end{array}$ \\
\hline $\begin{array}{l}\text { Vidéo vécu de } \\
\text { Romain en position } \\
\text { pause } \\
\text { Située à la } 35^{\text {ème }} \\
\text { minute }\end{array}$ & $\begin{array}{l}\text { Ah là, il a une posture qui est contre le tableau, il s'appuie comme s'il avait besoin de, } \\
\text { de soutien un petit peu. Et ça, on m'avait fait la remarque l'an dernier quand j'avais fait } \\
\text { le stage, j'avais un petit peu cette attitude-là (...) C'était une heu, formatrice à l'IUFM } \\
\text { oui, qui était venue nous visiter et elle m'avait fait cette remarque-là. Heu, ben moi, } \\
\text { j'avais pas forcément conscience que ça renvoyait cette image-là, parce que pour moi } \\
\text { non, j'attendais pas le soutien, je, j'attendais en fait. C'était pas pour moi du soutien, } \\
\text { etc. Mais ça peut effectivement renvoyer cette image-là, heu, le fait de... }\end{array}$ \\
\hline $\begin{array}{l}\text { Située à la } 58^{\text {ème }} \\
\text { minute }\end{array}$ & $\begin{array}{l}\text { Chercheur: qu'est-ce que tu as retenu ou quelles pistes [penses]-tu... } \\
\text { éventuellement [explorer]? } \\
\text { C'est plutôt sur ma pratique à moi que je vais un petit peu modifier justement et } \\
\text { essayer de réfléchir à modifier, c'est, heu, ben lorsque j'attends le silence, c'est } \\
\text { vraiment circuler un peu plus dans la salle... }\end{array}$ \\
\hline
\end{tabular}

Tableau 3 : Protocole à 2 volets discontinu pour pister une transformation projetée

\section{Table 3: Two-facetted discontinuous protocol to track a projected transformation}

Nous présentons ci-dessous l'analyse locale de la structure significative relative à la première transformation projetée de son activité en classe «Circuler dans les rangs pendant le rituel de début du cours ».

Unité d'expérience (elle correspond à la fraction de l'activité préréflexive qui est racontée, montrée ou commentée): Imagine une alternative à tester dans sa classe qui intègre des pistes issues à la fois des propos de la collègue d'EPS pour que les élèves se mettent plus rapidement en position scolaire et qui est renforcée par une autoréflexion sur sa propre manière de faire grâce au rappel d'un ancien feed-back formatif.

Représentamen (R) (il correspond à ce que l'acteur prend en compte, au temps t, dans la situation, pour agir en fonction de sa structure d'attente) :

— Les commentaires de l'enseignante d'EPS sur l'attitude les bras croisés (vidéo commentaires néotitulaire Aude) ;

— Les élèves perturbateurs de son propre rituel de classe (souvenir d'expériences de classe) ;

— L'image fixe de Romain adossé au tableau (vidéo entretien vécu de Romain) ;

— Le rappel d'une attitude adoptée comparable à celle de Romain (souvenir d'un retour formatif en stage).

Préoccupations (eR) (elles traduisent ce que cherche à faire l'acteur compte tenu de ce qui s'impose à lui dans la situation) :

— Trouver une piste pour gérer d'emblée les élèves perturbateurs

— Comparer les arguments d'Aude (prof. d'EPS) relatifs à l'attitude «les bras croisés» avec les siens

— Réinterpréter le sens de la remarque d'une formatrice 
— Envisager la situation du point de vue des élèves

Référentiel (sR) (il correspond aux connaissances construites dans des expériences passées et mobilisées à cet instant) :

- Pour que l'attitude «les bras croisés » soit efficace, il est nécessaire de balayer la classe du regard ou de regarder précisément les élèves qui ne sont pas en place

— Face à des élèves dans la lune qui oublient de sortir leurs affaires ou qui s'assoient avant que je le leur aie demandé, je leur rappelle

Interprétants (ils traduisent la présence dans l'activité ici et maintenant d'éléments de validation de cours d'action passés ou de construction de types nouveaux)

Validation du type :

— Rester immobile complètement et figé au niveau du regard n'intimide pas les élèves

- Construction des types :

— Je n'avais pas forcément conscience que ça renvoyait cette image-là (bras croisés adossé au tableau)

— Parce que finalement, c'est comme s'il s'effaçait un petit peu là, de, de s'appuyer au tableau

- Bouger dans la classe pour justement montrer aux élèves qu'ils ne sont pas en place, les faire s'activer et se mettre plus rapidement en position de travail.

Les éléments qui ont fait signe dans la situation de navigation et qui ont contribué à élaborer cette transformation «lorsque j'attends le silence, c'est vraiment circuler un peu plus dans la salle » sont issus à la fois des ressources de la plateforme (propos du professeur d'EPS et image de l'enseignant en difficulté) et des expériences de classe et de formation de l'enseignante que cela réveille (rappel d'élèves perturbateurs et rappel de sa propre attitude pointée par une formatrice). Ce qui a fait signe tout d'abord, ce sont les propos d'Aude qui relate que si cela lui arrive de prendre cette position les bras croisés, ce n'est jamais dans une attitude figée comme celle de Romain «Donc, ce que je fais moi c'est... je peux être dans cette position et me déplacer ou alors balayer du regard la classe et fixer un élève, par exemple celui qui parle pour essayer de le calmer ou éventuellement me placer à côté d'un élève qui justement bavarde ». Ces propos ont fait écho à son expérience de classe "comme elle dit de balayer la classe du regard ou alors, moi je m'attarde aussi sur heu, enfin je regarde précisément les élèves qui ne sont pas en place pour moi » et l'ont amenée à revoir et décrire les comportements inattentifs ou déviants de certains de ses élèves. La comparaison des façons de faire d'Aude avec sa propre pratique lui fait prendre conscience que s'il y a des aspects partagés, elle ne se déplace pas dans cette phase précise et délicate d'attente du silence et qu'il y a sans doute une piste à exploiter «ce que je pense que je vais heu, je vais faire, c'est effectivement ben passer dans la classe ». Cette piste est renforcée par la conscientisation de l'organisation spatiale particulière de sa classe qui ne favorise pas le contrôle des élèves uniquement par le regard «Oui parce qu'en plus, moi j'ai un bureau qui est complètement, il n'est pas au centre, il est tout à droite de la classe, bon avec un ordinateur et c'est vrai que je suis un peu en retrait finalement ».

Si ce premier extrait de séquence lui fait prendre conscience de la pertinence du déplacement dans cette phase du rituel, la suite de cette séquence discontinue dix minutes plus tard la focalise sur les effets d'une attitude figée adossée au tableau. L'image fixe de Romain dans la vidéo lui rappelle soudain, sous forme de flash, le retour d'une formatrice lors d'une visite de classe «on m'avait fait la remarque l'an dernier quand j'avais fait le stage, j'avais un petit peu cette attitude-là ». Si elle n'avait pas donné trop de sens à cette remarque à cette époque, c'est qu'elle ne voyait pas les effets potentiels que cette attitude pouvait générer chez les élèves. Elle en prend conscience lors de cette première session de navigation «finalement, c'est comme s'il s'effaçait un petit peu là, de, de s'appuyer au tableau » en voyant les comportements des élèves qui agissent comme s'il n'y avait pas de professeur et 
en le reliant aux propos d'Aude "mais rester immobile complètement et figé au niveau $d u$ regard, en fait, je pense que ça les intimide pas en fait et ils sentent qu'on est un peu en retrait et pas présent ». Cette exploration a contribué à renforcer la force de conviction de la première piste énoncée de se déplacer et à l'évoquer en bilan de la navigation commentée comme la première piste qu'elle expérimentera «c'est vraiment circuler un peu plus dans la salle».

\section{2.- Tensions professionnelles et pistes de transformation révélées via les ressources de la plateforme}

L'analyse des trois cours d'expérience en contexte d'autoformation et étalés dans le temps via la plateforme de vidéo formation a permis de reconstruire l'évolution des préoccupations de l'enseignante ainsi que les tensions professionnelles vécues en fonction de l'enrichissement de l'expérience professionnelle en classe (Tableau 5). Les tensions ont été identifiées via l'explicitation par Coralie d'engagements contradictoires révélés par la comparaison régulière de ce que font ou disent les enseignants à l'écran avec ses propres expériences de classe. Elles ont été repérées dans les données par l'identification d'oppositions récurrentes entre deux préoccupations et/ou attentes saillantes dans un faisceau de préoccupations (e.g. La préoccupation typique de Coralie de «préserver un niveau élevé de silence dans sa classe » s'oppose à la préoccupation de «mettre rapidement les élèves en activité ». Cette deuxième préoccupation constitue une piste de transformation de son activité mais elle risque produire, de son point de vue, un niveau sonore plus élevé s'opposant à la première). Ces tensions sont mises à jour grâce à la conscientisation de ses propres façons de faire (notamment en décrivant ses préoccupations-attentes en lien avec des épisodes de classe effectifs) et la comparaison à d'autres façons de faire (verbalisation de convergences et divergences) via des vidéos signifiantes pour elle, car liées à ses préoccupations professionnelles du moment.

Les premières préoccupations relatives à la période d'octobre, après un mois de classe, sont focalisées sur la gestion collective de l'entrée en classe, sur sa propre manière d'agir en lien avec la question de l'autorité et sur son développement professionnel. Au mois de décembre, elles ont évolué vers l'affinement du rituel de mise au travail des élèves avec la recherche d'une entrée disciplinaire plus rapide, l'anticipation-gestion de comportements perturbateurs de certains élèves et une réflexion sur l'usage de soi dans des situations difficiles. Les préoccupations à la fin du mois de janvier sont relatives à l'apprentissage des élèves, à la différenciation et aux modalités de travail proposé (à la maison et en classe). On peut noter une évolution des préoccupations centrées, au début de l'année, sur le collectif classe et sur soi-même vers des préoccupations focalisées, au bout de 6 mois d'expérience, sur les élèves et la prise en compte de leurs difficultés individuelles. Il est intéressant de noter que chacune de ces préoccupations s'actualise à travers diverses amorces contenues dans la plateforme (à travers les titres des vidéos des thèmes 1 et 2) et que l'explicitation de ses propres pensées à un interlocuteur permet de révéler les tensions professionnelles vécues par cette enseignante qui sont la plupart du temps partagées par la communauté d'enseignants. Ces différentes problématisations situées dans le temps scolaire ont toutes débouchées sur l'auto-élaboration par l'enseignante d'une ou plusieurs pistes de transformations projetées au niveau de sa propre pratique en classe (Tableau 4). Ces auto-transformations projetées possèdent plus ou moins de force de conviction et leur mise en œuvre, quand elle est évoquée, est envisagée dans des temporalités plus ou moins proches. 


\begin{tabular}{|c|c|c|}
\hline $\begin{array}{l}\text { Thèmes et } \\
\text { périodes de } \\
\text { l'année }\end{array}$ & $\begin{array}{l}\text { Tensions professionnelles } \\
\text { construites }\end{array}$ & $\begin{array}{c}\text { Auto-transformation projetée en lien avec ses } \\
\text { propres classes }\end{array}$ \\
\hline $\begin{array}{l}\text { Début de cours } \\
\text { Oct. } 2011\end{array}$ & $\begin{array}{l}\text { Attendre le calme pour faire s'asseoir } \\
\text { les élèves vs démarrer plus vite en } \\
\text { mettant les élèves en activité }\end{array}$ & $\begin{array}{l}\text { Circuler dans les rangs pendant le rituel } \\
\text { Ne pas rester adossé au tableau }\end{array}$ \\
\hline $\begin{array}{l}\text { Autorité } \\
\text { Oct. } 2011\end{array}$ & $\begin{array}{l}\text { Imposer le silence vs mettre les } \\
\text { élèves au travail rapidement en } \\
\text { acceptant un niveau sonore plus élevé }\end{array}$ & $\begin{array}{l}\text { Démarrer une séance directement avec le } \\
\text { vidéoprojecteur pour capter l'attention et } \\
\text { mobiliser les élèves }\end{array}$ \\
\hline $\begin{array}{l}\text { Développement } \\
\text { professionnel } \\
\text { Oct. } 2011\end{array}$ & $\begin{array}{l}\text { Construction progressive et } \\
\text { permanente vs tout se joue à des } \\
\text { périodes-clés (Noël, première année) }\end{array}$ & $\begin{array}{l}\text { Confortée dans son point de vue initial d'un } \\
\text { développement professionnel progressif }\end{array}$ \\
\hline $\begin{array}{l}\text { Rituel de mise au } \\
\text { travail } \\
\text { Déc. } 2011\end{array}$ & $\begin{array}{l}\text { Installer le rituel en silence vs } \\
\text { autoriser le dialogue entre les élèves }\end{array}$ & $\begin{array}{l}\text { Interdire aux élèves }\left(6^{\text {ème }}\right) \text { de poser leur cartable } \\
\text { sur le dossier de leur chaise (source de } \\
\text { bavardage, perturbations) }\end{array}$ \\
\hline $\begin{array}{l}\text { Mise au travail } \\
\text { dans sa discipline } \\
\text { Déc. } 2011\end{array}$ & $\begin{array}{lll}\text { Vérifier les } & \text { exercices } \\
\text { individuellement vs } & \text { contrôler } \\
\text { collectivement la classe } & \end{array}$ & $\begin{array}{l}\text { Envoyer plusieurs élèves au tableau }\left(4^{\text {ème }}\right) \text { en } \\
\text { janvier (nécessité de réfléchir les exercices et } \\
\text { d'anticiper les réactions perturbatrices d'élèves) }\end{array}$ \\
\hline $\begin{array}{l}\text { Sanction } \\
\text { Déc. } 2011\end{array}$ & $\begin{array}{l}\text { Intervenir } \\
\text { d'élèves perturbateurs vs gérer } \\
\text { d'agitation au niveau du collectif } \\
\text { classe }\end{array}$ & $\begin{array}{l}\text { Prévoir des exercices pour un climat de début de } \\
\text { cours agité, mais force de conviction de la } \\
\text { proposition limitée (sentiment d'injustice pour les } \\
\text { élèves sérieux) }\end{array}$ \\
\hline $\begin{array}{l}\text { Usage de soi } \\
\text { Déc. } 2011\end{array}$ & $\begin{array}{l}\text { S'énerver face à une classe agitée } v s \\
\text { s'apaiser soi-même pour apaiser les } \\
\text { élèves }\end{array}$ & $\begin{array}{l}\text { Désamorcer dès l'entrée en classe des attitudes } \\
\text { conflictuelles par un petit mot } \\
\text { Clore les discussions des élèves de manière plus } \\
\text { douce }\end{array}$ \\
\hline $\begin{array}{l}\text { Apprentissage } \\
\text { Janv. } 2012\end{array}$ & $\begin{array}{l}\text { Aider les élèves à travailler et à } \\
\text { apprendre } v s \text { trop les guider }\end{array}$ & $\begin{array}{l}\text { Proposer un travail de recherche sur un problème } \\
\text { en classe comme alternative aux devoirs à la } \\
\text { maison }\end{array}$ \\
\hline $\begin{array}{l}\text { Différenciation } \\
\text { Janv. } 2012\end{array}$ & $\begin{array}{l}\text { Aider les élèves en difficulté } v s \\
\text { avancer le cours }\end{array}$ & $\begin{array}{l}\text { Proposer des formes de défi par groupes } \\
\text { (difficulté de gérer les décalages entre élèves) }\end{array}$ \\
\hline $\begin{array}{l}\text { Fin de cours } \\
\text { Janv. } 2012\end{array}$ & $\begin{array}{l}\text { Donner le travail à faire à la maison } \\
v s \text { finir son cours }\end{array}$ & $\begin{array}{l}\text { Noter les devoirs au fil du cours sur un coin du } \\
\text { tableau dédié à cela, mais force de conviction de } \\
\text { la proposition limitée (car pas de consensus } \\
\text { professionnel) }\end{array}$ \\
\hline
\end{tabular}

Tableau 4 : Synthèse des principaux problèmes professionnels explorés et des pistes de transformations envisagées. (Chaque couleur différente représente ce qui a été construit dans une session de navigation à une période particulière de l'année)

Table 4: Overview of the reconstruction of the main professional tensions experienced and possible changes envisaged. (Each different color represents what was built in a browsing session at a particular time of year)

\section{3.- Suivi des transformations projetées en situation de classe, sources d'influence et effets}

Le suivi de quatre transformations construites à partir des explorations de la plateforme a débouché sur l'expérimentation de trois d'entre elles en classe. Ces expérimentations nouvelles pour l'enseignante dans sa classe se sont appuyées sur des apprentissages professionnels anticipés via les ressources vidéo de la plateforme à partir d'une mise en lien systématique avec ses propres expériences professionnelles qui faisaient écho à la situation. Les expérimentations que cela a suscitées en classe ont donné lieu à de nouveaux apprentissages à partir de leur mise en œuvre effective et de l'explicitation des effets repérés sur sa propre activité et celle des élèves. Nous présentons premièrement le suivi d'une piste de transformation projetée en situation de classe, de sa mise en place à sa mise en œuvre et deuxièmement une vision synthétique des pistes de transformations et de leurs différentes sources d'influence. 
L'élaboration de la piste de transformation visant une mise au travail plus rapide dans sa discipline a été initiée à partir d'un double représentamen, l'un mnémonique issu de son expérience "c'est avec les 4 e où j'ai un petit peu plus de problèmes de discipline et de bruit de fond» et l'autre relatif à l'environnement à travers le titre de la vidéo qui joue un rôle d'attracteur «règles économiques ${ }^{14}$ ». Ces deux representamen ont permis de spécifier son engagement afin d'élaborer un rituel adapté pour sa classe de $4^{\text {ème }}$ "c'est vrai que les $4 e$, je ne le fais pas ça (rituel des $6^{\text {ème }}$ ) parce que bon, ils étaient aussi, enfin je les trouvais un peu grands pour ce type de rituel. Mais il faudrait que peut-être j'en trouve d'autre ». La piste de transformation projetée a pris naissance à partir de l'identification d'un décalage entre ce que perçoit l'enseignante de la pratique visionnée ou évoquée d'un pair (débutante) et sa propre pratique «c'est vrai qu'elle gagne (du temps), enfin, moi la vérification des exercices, je trouve que ça me fait perdre du temps ». Ce décalage est problématisé en relation avec des exigences professionnelles et révèle une tension dans la pratique "C'est nécessaire hein parce que voilà il faut quand même qu'on vérifie que les élèves ils aient fait leurs exercices, mais ça, pendant ce temps, les autres ils en profitent pour bavarder». Cette tension «Vérifier les exercices individuellement $v s$ contrôler collectivement la classe » s'exprime à travers l'évocation de l'expérience personnelle «quand je passe dans une rangée, ceux de l'autre rangée ben ils en profitent pour bavarder. Donc, je me retourne, je précise que voilà, on est en classe et qu'on arrête les bavardages. Mais c'est vrai que c'est sans cesse, à chaque cours ». Cette immersion mimétique conduit l'enseignante à trouver dans la façon de faire de l'enseignante filmée une piste de transformation "c'est une bonne idée d'envoyer trois élèves au tableau pour faire ben un petit, un petit entraînement $»$. Le caractère habituel de cette façon de faire pour débuter le cours qu'elle repère chez l'enseignante renforce son intérêt puisqu'elle est à la recherche d'un rituel adapté pour sa classe de $4^{\text {ème }}$. Elle cherche rapidement à imaginer comment la concrétiser avec ses propres classes à travers des unités d'expérience anticipées «ça peut être du calcul mental pour les 6e. Parce que bon, on est, ou un petit exercice» et «les 4e pareil, peut-être des, des, même des points de chapitres qu'on a déjà traités, qui ont peut-être du mal à rentrer, pour ben peut-être y revenir à chaque début d'heure». L'expérimentation en classe validera les effets attendus non seulement en termes de gain de temps, mais aussi en contrôle du collectif classe à travers la construction des types «c'est vrai que ça permet déjà d'aller plus vite dans la correction » et "quand je passe dans les rangs, ils sont occupés à regarder ce que les camarades font et à comparer avec leur production à eux». Les effets sur sa propre activité sont moins coûteux que ce qu'elle avait imaginé (anticiper des exercices et des réactions perturbatrices d'élèves au tableau) «ça ne m'a pas demandé plus de travail... ça me demande juste cinq minutes avant d'écrire les énoncés au tableau. Si éventuellement je n'ai pas le temps, je le leur fais écrire au moment où ils corrigent». Une difficulté pointée par l'enseignante concerne les contenus disciplinaires que cela permet d'aborder (faciles pour des exercices numériques et un peu plus difficiles en géométrie). Cette difficulté a été résolue en partie après un échange avec sa tutrice «elle m'a donné des pistes pour corriger les exercices de géométrie avec, notamment, sur transparent, en fait, pareil, projeter la solution aux élèves sur transparent ». Les expressions utilisées par l'enseignante en entretien expriment la force de conviction attachée à cette transformation "c'est une bonne idée », "ça peut-être pas mal», «j'aurais dû le regarder avant » et après l'avoir expérimenté en classe « ça, je continue ».

Toutes les pistes de transformations projetées se sont construites en articulant des extraits significatifs de vidéos de la plateforme pour l'enseignante avec l'explicitation de sa propre

14 Cet extrait vidéo « met en avant des modalités d'accueil des élèves de plus en plus structurées. Les enseignants anticipent très tôt l'entrée des élèves, organisent un « sas d'accueil » en montrant physiquement et symboliquement le changement de territoire, la frontière entre les couloirs et le lieu de travail (...). Une série d'activités « routinisées » mobilisent très tôt l'attention des élèves (...) et intègrent de manière indissociable une mise en situation des apprentissages et un encadrement des comportements » (Ria et collaborateurs, 2010). http://neo.ens-lyon.fr/neopass/index.php?themes=1\&activites=7 
expérience en écho (Tableau 5). Les propos d'une formatrice ou d'une tutrice ont constitué les autres sources d'influence qui ont contribué à renforcer ou à diminuer l'intérêt des pistes construites.

\begin{tabular}{|c|c|c|c|}
\hline $\begin{array}{l}\text { Auto-transformation } \\
\text { projetée }\end{array}$ & $\begin{array}{l}\text { Sources d'influence } \\
\text { liées à la plateforme }\end{array}$ & $\begin{array}{l}\text { Autres sources } \\
\text { d'influence }\end{array}$ & $\begin{array}{l}\text { Auto-transformation réalisée } \\
\text { ou non et effets }\end{array}$ \\
\hline $\begin{array}{l}\text { Circuler dans les } \\
\text { rangs pendant le rituel } \\
\text { Ne pas rester adossé } \\
\text { au tableau }\end{array}$ & $\begin{array}{l}\text { Vidéo commentaires } \\
\text { professeur d'EPS } \\
\text { débutante (thème 1) } \\
\text { Vidéo vécu Romain } \\
\text { professeur d'Allemand } \\
\text { débutant (thème 1) }\end{array}$ & $\begin{array}{l}\text { Expérience } \\
\text { professionnelle } \\
\text { d'élèves } \\
\text { perturbateurs } \\
\text { Retour formatrice } \\
\text { IUFM en stage }\end{array}$ & $\begin{array}{l}\text { Réalisé } \\
\text { Gain en temps et en calme, } \\
\text { élèves plus vite au travail } \\
\text { Micro-gestes professionnels } \\
\text { construits: «trouver le } \\
\text { regard des élèves », «lui } \\
\text { montrer que je le vois» }\end{array}$ \\
\hline $\begin{array}{l}\text { Démarrer une séance } \\
\text { directement avec le } \\
\text { vidéoprojecteur pour } \\
\text { capter l'attention et } \\
\text { mobiliser les élèves } \\
\left(4^{\text {ème }}\right)\end{array}$ & $\begin{array}{l}\text { Vidéo de classe } \\
\text { Romain } 6 \text { mois plus } \\
\text { tard (thème } 1 \text { ) }\end{array}$ & $\begin{array}{l}\text { Expérience } \\
\text { professionnelle } \\
\text { d'utilisation du } \\
\text { vidéoprojecteur } \\
\text { avec des élèves } \\
\left(6^{\text {ème }}\right) \\
\text { Propos-freins } \\
\text { tutrice }\end{array}$ & $\begin{array}{l}\text { Non réalisé } \\
\text { Car avis nuancé de la tutrice } \\
\text { En attente d'un contexte } \\
\text { favorable }\end{array}$ \\
\hline $\begin{array}{l}\text { Interdire aux élèves } \\
\left(6^{\text {eme }}\right) \text { de poser leur } \\
\text { cartable sur le dossier } \\
\text { de leur chaise (source } \\
\text { de perturbations) }\end{array}$ & $\begin{array}{l}\text { Vidéo de classe } \\
\text { professeur de maths } \\
\text { débutante (thème 1) }\end{array}$ & $\begin{array}{l}\text { Expérience } \\
\text { professionnelle } \\
\text { des } \\
\text { retournements et } \\
\text { bavardages } \\
\text { récurrents }\end{array}$ & $\begin{array}{l}\text { Réalisé } \\
\text { Empêche de bavarder ou en } \\
\text { limite la possibilité }\end{array}$ \\
\hline $\begin{array}{l}\text { Envoyer plusieurs } \\
\text { élèves au tableau } \\
\left(4^{\text {ème}}\right) \text { en janvier } \\
\text { (réfléchir les exercices } \\
\text { et anticiper les } \\
\text { réactions } \\
\text { perturbatrices) }\end{array}$ & $\begin{array}{l}\text { Vidéo de classe } \\
\text { professeur de maths } \\
\text { débutante (thème 1) }\end{array}$ & $\begin{array}{l}\text { Expérience } \\
\text { professionnelle } \\
\text { de la difficulté } \\
\text { d'avoir le silence } \\
\text { lorsqu'on passe } \\
\text { dans les rangs }\end{array}$ & $\begin{array}{l}\text { Réalisé } \\
\text { Gain de temps dans la } \\
\text { correction et attention } \\
\text { focalisée sur le travail des } \\
\text { camarades au tableau (facile } \\
\text { pour le numérique, mais plus } \\
\text { difficile en géométrie) } \\
\text { Retour-aide tutrice pour } \\
\text { construire une piste en } \\
\text { géométrie }\end{array}$ \\
\hline
\end{tabular}

Tableau 5 : Synthèse des auto-transformations projetées et expérimentées et des principales sources d'influence. (Chaque couleur différente représente ce qui a été construit dans une session de navigation à une période particulière de l'année)

Table 5: Summary of projected and tested self-transformations and major sources of influence. (Each different color represents what was built in a browsing session at a particular time of year)

\section{5.- Discussion générale}

Nous avons montré que les navigations dans la plateforme de vidéo formation sont vécues comme des expériences mimétiques actives qui mettent en œuvre des processus de projections-identifications multiples permettant non seulement de mobiliser et d'interroger des connaissances professionnelles et des expériences passées, mais également d'en construire de nouvelles. La confrontation d'enseignants débutants à cet environnement de vidéo formation génère une double activité de perception-simulation et d'imaginationprojection que nous rattachons à deux concepts-clés chez Simondon $(2005,2008)$ : la «résonance interne» qui permet de faire interagir deux ordres de réalités différentes et «l'imagination » qui correspond à l'élaboration d'images mentales conscientes ou non et qui possèdent une force «pro-active ». Cette activité imaginative qui est une réelle anticipation de l'acte en cours de développement s'actualise aussi bien en amont de la perception, 
pendant celle-ci qu'après la réalisation d'une action. La configuration typique des navigations des formés à travers un «visionnement de la vidéo de classe » suivi de l'accès au «vécu professionnel de l'enseignant» articule ces deux processus de résonance-imagination. Cette articulation ne se limite pas à la reprise des aspects de surface liés aux comportements manifestes de l'enseignant filmé. Durant l'observation de la vidéo de classe, le voir est un voir «en écho» et non un voir pour «imiter des aspects formels et superficiels» du comportement de l'enseignant. Il se synchronise toujours avec des connaissances, expériences de classe vécues plus ou moins proches de la situation visionnée qui sont mises en relation et qui permettent de créer des échanges interactifs de type mimétiques (attitudes, gestes, déplacements, communications, silences, intonations...) entre l'enseignant se formant et l'enseignant à l'écran.

Dans le cadre de notre approche théorique enactive (Varela, 1989; Varela, Thompson, \& Rosch, 1993), les processus d'apprentissage-développement a) sont considérés comme inhérents à toute activité humaine et donc accompagnent la dynamique des couplages acteurenvironnement de manière permanente dans des situations de travail et d'apprentissageformation instituées ou non, b) sont appréhendés du point de vue de l'organisation du système lui-même en relation avec son environnement, c) sont envisagés à partir d'un couple modification/continuation traduisant la processivité et l'inscription dans différentes temporalités, d) impliquent l'acteur dans sa totalité c'est-à-dire dans ses dimensions cognitives, interprétatives, imaginatives, créatives, émotionnelles, corporelles et sociales. Nous développons cette réflexion en l'articulant avec la théorie «allagmatique » développée par Simondon qui consiste en une "théorie générale des échanges et des modifications des états » ou "une théorie générale des transformations» s'appliquant aussi bien au niveau physique, biologique et psychique que social. En refusant de faire de l'individu une réalité donnée, substantielle et statique, cette théorie se focalise sur les processus d'individuation comme le devenir, la relation, les opérations et l'ontogenèse. Dans notre approche théorique, l'apprentissage-développement est appréhendé sous la forme d'un couple inséparable dans lequel les deux notions, difficilement dissociables, se co-déterminent.

\section{1.- L'apprentissage-développement comme concaténation d'expériences multiples sur des temporalités différentes}

L'apprentissage-développement est permanent et accompagne l'activité quotidienne sous la forme d'une dérive «chaotique » peu prédictible et dont l'explication ne peut se donner de manière causaliste (Durand, 2008) compte tenu du nombre d'interactions possibles entre les différentes expériences vécues par les acteurs et de la construction du sens qu'ils peuvent en donner. L'apprentissage-développement ne peut donc être envisagé comme la conséquence de la transmission d'une représentation adéquate d'un monde extérieur prédéterminé qu'il faudrait réduire et reconstituer dans une situation de formation. Nous l'appréhendons plutôt de manière plurielle et complexe comme un dialogue permanent entre acteur-environnementautrui «à travers la concaténation, sur des périodes de temps plus ou moins longues, de l'action, de la planification, de la réflexion située et de l'observation de l'action des autres et de réception de l'apport didactique des autres » (Theureau, Durand, Leblanc, Saury, \& Sève, 2004 , p. 8). De notre point de vue, apprendre ne peut donc se réduire à s'approprier des savoirs, des règles prédéfinies et prescrites par un formateur dans un contexte de formation institutionnalisé, mais consiste à «faire-émerger» sur la base des différentes expériences vécues des questions dont la pertinence est dictée par les significations construites par l'acteur lui-même dans un «environnement capacitant ${ }^{15} »$. Ce questionnement produit à partir de réalités disparates issues de son vécu et des ressources de l'environnement favorise

15 Un « environnement capacitant » est conçu pour favoriser la mise en œuvre effective des capacités des acteurs. Les concepteurs de ces environnements cherchent à déterminer les conditions favorables et les «facteurs de conversion » du potentiel d'action des acteurs en possibilité réelle (Falzon, 2013). 
l'acquisition par apprentissage «de modes d'action nouveaux issus du patrimoine culturel professionnel en relation ou non avec un projet intentionnel de transmission par un formateur de ces modes d'action » (Durand, 2008, p. 100). Dans notre étude, l'acteur autoconstruit de nouvelles façons d'agir, en dehors d'un projet de transmission précis d'un formateur, en tirant-bénéfice à la fois de la structuration des ressources vidéo qui a été pensée à partir d'un modèle d'apprentissage-développement de l'activité (e.g. à l'échelle d'un même enseignant et/ou de la communauté enseignante débutante) et en même temps de la modalité d'alloconfrontation lui permettant d'expliciter ses pensées et projets de transformation.

Ces apprentissages «ici et maintenant» s'articulent avec le développement du sujet que nous définissons à un niveau typique-générique et sur du temps plus long comme «la transformation de son répertoire d'actions" (Durand, 2008, p. 100). Pour saisir et comprendre le développement du sujet, c'est-à-dire les éléments qui génèrent des changements ou au contraire des permanences dans son activité, nous nous appuyons sur la notion de «dispositions à agir » définies comme une «propension ou tendance de l'acteur à agir (ou réagir) d'une certaine manière dans des circonstances déterminées » (Lahire, 1998, p.95). Si l'activité se caractérise par des transformations permanentes, elle montre aussi «des régularités et des redondances qui peuvent être désignées comme des expressions identitaires d'un sujet et d'un environnement relativement stable » (Durand, 2008, p. 115). La stabilité des dispositions à agir serait due au fait que d'une part les conditions structurelles qui les ont produites se maintiennent et se perpétuent, sollicitant ainsi continuellement leur actualisation et d'autre part qu'elles font preuve d'une forme d'économie et d'efficacité pour les personnes qui les mobilisent. En confrontant les acteurs à des situations nouvelles, qui mettent en présence du «passé » et du «présent» différents, hétérogènes et pluriels, on contribue à révéler à la fois le "pouvoir négatif d'inhibition $d u$ passé» (Lahire, 1998), de refoulement d'une partie des dispositions à agir liées aux situations antérieures et en même temps d'appréhender le potentiel d'activation de compétences, de manière de dire et de penser, laissées à l'état de veille jusqu'à ce jour (Leblanc, sous presse). En faisant réfléchir à l'efficacité professionnelle sous l'angle de sa double valence «objective » et «subjective » (Saujat, 2010), on favorise la recherche de compromis entre les normes personnelles de viabilité des acteurs en relation avec la recherche de confort, d'efficience et de plaisir professionnels au niveau de l'usage de soi et les normes prescrites par l'institution en relation avec les objectifs de performance (Falzon, 2013 ; Ria, 2009 ; Saujat, 2010). La formation gagnerait à prendre en compte sérieusement l'articulation du couple apprentissage-développement en s'intéressant à la fois aux apprentissages réalisés in situ ici et maintenant et en même temps au développement appréhendé à travers le suivi longitudinal des trajectoires professionnelles.

Cette vision de l'apprentissage-développement s'accorde avec la théorie allagmatique développée par Simondon (2005) qui définit dans un sens plus large les processus d'individuation humaine aux niveaux biologique, psychique et social. L'individuation est une "opération qui fait advenir de l'individué à partir du préindividuel, du déterminé à partir de l'indéterminé » (Château, 2008, p. 49) en produisant des différenciations et des formes individualisantes toujours chargées de potentiel permettant de rendre infini ce processus de transformations futures. Pour Simondon, les individus ne sont que des «phases momentanées » de ce processus d'autoconstitution qui ne peut ni être imposé de l'extérieur ni suivre une trajectoire déterminée. Pour envisager toute réalité non pas seulement comme structure, mais comme être s'individuant, comme relation, opération, l'individuation doit «être saisie comme devenir de l'être, et non comme modèle de l'être qui en épuiserait la signification » (Simondon, 2005, p. 31).

Dans notre perspective, étudier l'apprentissage-développement revient à s'intéresser aux transformations du flux de l'activité qui «traversent» les différentes situations de formation et de pratiques professionnelles quotidiennes afin d'identifier et de comprendre les 
potentialités nouvelles qui s'y ouvrent, s'y ferment, s'y actualisent et s'y construisent. Ainsi, l'apprentissage-développement est aussi présent en situation professionnelle ${ }^{16}$ et il est intéressant d'étudier comment l'activité «s'étale » dans le «milieu associé » de l'individu, qui n'est pas un milieu général, indifférencié, objectif, mais correspond au «monde propre » effectif pour l'individu, découpé et reconstruit dans l'environnement objectif par ses attentes et ses capacités propres. Comprendre l'individu consiste donc à décrire la genèse de l'individuation de l'individu, mais aussi de son milieu associé au lieu de le présupposer ou de le prédéterminer. À travers nos travaux, nous soutenons l'hypothèse que les environnements de vidéo formation conçus à partir de l'analyse du travail effectif d'enseignants constituent un vecteur d'individuation psychique, collective et technique en favorisant l'établissement de relation entre le «je» et le «nous» à travers le milieu associé concret et effectif qu'ils permettent de construire et l'adressage d'un discours à autrui (chercheur, formateur, formé). Une condition de cette mise en relation est de trouver un rapport proximité/distance satisfaisant entre «ce qui est donné à voir »/« vécu du spectateuracteur-se formant» qui soit en phase avec ses dispositions à agir du moment. Ce rapport proximité/distance peut-être appréhendé également en termes d'intégration communautaire, c'est-à-dire d'intégration de l'individu au social, à travers un double processus de «transindividualité » définit comme l'individuation ${ }^{17}$ passant par le collectif (Simondon, 1989).

\section{2.- Transindividualité entre «l'in-group » des novices et «l'out-group» des expérimentés}

Ce processus se réalise soit de manière analogique à travers l'activité novice dans la mesure où le passé et l'avenir des uns et des autres coïncident assez fortement, soit de manière non analogique à travers l'activité des chevronnés dans laquelle les novices ne se reconnaissent pas d'emblée (Ria, \& Leblanc, 2012). Le premier processus de «transindividualité » entre novices permet de se reconnaître comme appartenant à une communauté apprenante en cours de construction et d'entrevoir des perspectives de progrès réalistes et atteignables au vu de leurs dispositions à agir du moment. Cette communauté correspond à un "groupe d'intériorité » ou «in-group» qui peut être considéré comme le corps social ou la personnalité sociale du sujet (ibid.). Les agissements de leurs pairs dans les vidéos de classe constituent des sortes de «preuve » du caractère réalisable pour des enseignants débutants dans le métier. Ce processus non pensé et souvent écarté de la formation initiale est dans l'environnement de vidéo formation Néopass@ction non seulement possible, mais valorisé par la conception même des ressources et de leur agencement. La navigation prioritairement orientée en direction d'autres néotitulaires les aide à la fois à se reconnaître, se rassurer et à renforcer le sentiment d'appartenance professionnelle qui leur fait tant défaut en début de carrière et en même temps à trouver des pistes de transformation en prise avec leurs préoccupations et réalistes. "L'in-group est source de virtualités, de tensions, comme l'avenir individuel; il est réservoir de présence par ce qu'il précède l'individu dans la rencontre du "groupe d'extériorité" » (ibid., p. 178).

Le deuxième processus de «transindividualité » se déploie à travers les commentaires bienveillants, critiques et constructifs proposés par les enseignants plus anciens qui ont accepté d'être filmés à propos des situations professionnelles typiques des néotitulaires. Il peut contribuer à déplacer le regard égocentré des novices vers un point de vue allocentré.

16 Mais certaines conditions de travail peuvent limiter fortement ces apprentissages. Les situations de formation sont $a$ priori plus favorables à ces apprentissages à condition de trouver la bonne distance avec les situations réelles de travail.

17 Nous la définissons comme un processus de transformation de l'individu en tant que demeurant toujours inachevé et comme un processus d'objectivation de l'expérience, de la relation à soi-même et au monde extérieur (Simondon, 2005). 
Les commentaires des professionnels plus anciens articulant délimitation du problème professionnel, analyse des effets de telle ou telle façon de faire sur la situation et/ou analyse rétrospective de leur propre manière d'agir offrent aux novices un enrichissement possible de l'interprétation du couplage situation/enseignant, un gain en généralité ainsi que des pistes pratiques nouvelles. Pour les faire progresser vers ce point de vue en troisième personne, il est nécessaire de favoriser leur confrontation à des activités et des témoignages d'enseignants plus chevronnés à partir de mots-clefs ou d'artéfacts faisant sens par rapport à leurs propres expériences ou difficultés. Le social, nous dit Simondon, est constitué de la «médiation entre l'être individuel et le out-group» ("groupe d'extériorité»), mais «par l'intermédiaire de l'in-group» (ibid., p. 177). Ce qui signifie qu'il n'est pas pertinent d'opposer les deux groupes puisqu'ils sont en interactions, mais qu'il est plutôt intéressant d'envisager les conditions de leur coopération et du partage cognitif dans celle-ci (Saury, 2008). La notion de «contexte partagé » défini comme "un ensemble d'informations ou d'événements contextuels mutuellement manifestes pour un ensemble d'acteurs, à un instant t dans une situation donnée » (Salembier, \& Zouinar, 2004, p. 79) nous semble utile pour rendre compte d'une co-construction de cette intelligibilité mutuelle qui est reproduite dans la plateforme Néopass@ction et qui se prolonge dans la «mise en usage ». L'intelligibilité mutuelle est favorisée par un accès à l'activité «déconstruite et reconstruite » d'enseignants débutants qui fait l'objet de discussions professionnelles intergénérationnelles via la médiatisation des chercheurs-concepteurs. Ces discussions implémentées dans un environnement matériel, social et culturel particulier deviennent de véritables ressources disponibles à partager pour les différents acteurs (Salembier, Theureau, Zouinar, \& Vermersch, 2001). Comme "l'individu doit trouver une individuation sociale qui recouvre son individuation personnelle » (ibid., p. 177), on voit l'intérêt de pouvoir investir et comparer à travers cet environnement matériel plusieurs groupes au statut différent («ingroup » et «out-group »).

En amenant les chevronnés à aborder les problèmes professionnels à partir des préoccupations initiales des novices et en suscitant des chemins ou des parcours préférentiels dans la plateforme, on crée les conditions pour qu'un réel partage intergénérationnel puisse opérer. De plus, la pluralité des situations analysées ainsi que des acteurs impliqués dans cette enquête sur le travail réel (néotitulaires, chevronnés, chercheurs) fait de Néopass@ction le carrefour de nouvelles rencontres autour de la professionnalité enseignante avec de nouveaux espaces d'intéressement professionnel stimulant à la fois un processus de transmission de l'expérience, mais aussi un processus de création, d'innovation des pratiques pour s'adapter aux évolutions futures du métier. Cette intégration progressive dans une communauté de pratique, de la périphérie vers le centre ${ }^{18}$ (Lave, \& Wenger, 1991), avec des niveaux de légitimation différents, favorise l'émergence de formes de reconnaissance qui concourent par leurs interactions au processus d'individuation individuel et collectif.

\section{3.- Implications pratiques en termes de conception et d'accompagnement}

Pour saisir des phénomènes intéressants relatifs à l'apprentissage-développement professionnel, notre approche combine deux principes méthodologiques : celui de choisir des situations qui «jouent», pour les acteurs impliqués, sur l'opposition «familier/inhabituelle», et celui de «validité écologique» à partir de situations d'expérimentation qui respectent un isomorphisme partiel avec les situations culturelles observées (Theureau, \& Jeffroy, 1994). Compte tenu du caractère d'inachèvement de

18 Dans cette étude, sur 18 vidéos consultées par l'enseignante débutante : 11 vidéos concernent des débutants, 5 des expérimentés (dont 4 à la fin de la troisième session), et 2 des chercheurs. Ces chiffres renforcent les résultats d'une précédente étude qui avait pointé cette intégration progressive dans la communauté (Ria, \& Leblanc, 2012). 
l'homme et de son développement essentiellement culturel à travers les objets et systèmes techniques (Stiegler, 2010), il nous semble utile et fécond de concevoir et d'étudier des situations de formation instrumentées qui ouvrent des «espaces de possibles » pouvant paraître partiellement artificiels aujourd'hui, mais qui possèdent le potentiel de devenir le quotidien de demain.

Du point de vue de la conception de ces environnements de formation (e.g. Néopass@ction), il ne s'agit donc pas de prédire l'activité, mais plutôt d'approcher «les formes possibles de l'activité future » des utilisateurs dans la mesure où celle-ci ne peut pas être prévue dans le détail (Daniellou, 2007). À partir de l'anticipation partielle des attentes et préoccupations des futurs utilisateurs et d'un pari sur leurs intentions souhaitables, la «scénarisation médiatique » (Henri, Compte, \& Charlier, 2007) consiste à organiser un ensemble d'unités signifiantes de manière non linéaire s'éloignant d'une planification formalisée et détaillée de parcours de navigation qui seraient imposés aux utilisateurs (Hotte, Godinet, \& Pernin, 2007). La scénarisation consiste plutôt à créer des «Espaces d'Actions Encouragées » (Durand, 2008) offrant des possibilités d'apprentissage-développement ${ }^{19}$ des situations professionnelles à travers un dispositif que l'on peut qualifier de "propensionnel » (Jullien, 2009) en multipliant les points de vue et les portes d'entrées pour favoriser l'émergence de significations, celles-ci n'étant "pas enfermées à l'intérieur des symboles » (Winograd, \& Florès, 1989). Cette démarche de conception est à rapprocher de la notion «d'environnements capacitants» qui favorisent «la transformation d'une ressource potentielle (capacité) en ressource effective (capabilité)» (Six-Touchard, \& Falzon, 2013, p. 239). Un environnement capacitant constitué de ses seules ressources ne suffit pas pour créer du développement professionnel (Arnoud, J., \& Falzon, 2013). Il est donc nécessaire d'élaborer des dispositifs spécifiques pour faire interagir de manière fructueuse les ressources et les dispositions à agir des sujets avec celles de l'environnement capacitant afin d'identifier «les conditions de la possible conversion de ces ressources en réalisations concrètes » (ibid., p. 225). La méthode d'alloconfrontation vidéo (Mollo, \& Falzon, 2004 ; Leblanc, 2014b) utilisée comme une aide à l'explicitation et à l'accompagnement des transformations de l'activité du formé constitue un dispositif fécond pour révéler le potentiel formatif de cet environnement capacitant de vidéo formation. Ces dispositifs semblent particulièrement pertinents pour préparer à des tâches jugées complexes, composées d'objectifs ambitieux, lointains, définis par de multiples conditions et nécessitant une activité de conception importante de la part des acteurs. Ainsi la définition du travail prescrit pour les enseignants en termes de mission les oblige à disposer d'une marge de liberté pédagogique conséquente utile pour concevoir, définir, résoudre des problèmes et évaluer eux-mêmes les moyens de leur travail (Durand, 1996 ; Rogalski, 2003). L'intérêt de ces dispositifs est donc à nuancer en fonction des marges de manœuvre dont disposent les acteurs dans le cadre de l'organisation de leur travail.

Du point de vue de l'accompagnement, le caractère productif et profitable de ces situations d'alloconfrontation vidéo dépend largement de la qualité de la collaboration engagée dans les entretiens (verbalisations simultanées et interruptives) en permettant de dévoiler progressivement et de manière non-homogène les significations que les acteurs donnent à leurs commentaires et à leurs actions (dans la situation simulée et dans la situation de travail réel). L'attitude empathique de l'interlocuteur (chercheur, formateur ou autre formé),

19 Un «Espace d'Actions Encouragées » est porté à générer potentiellement les effets suivants : a) mettre en relation que tels ou tels aspects de l'activité de l'enseignant à l'œuvre et qui se déploient dans la situation de classe ont de bonnes chances d'engendrer tels effets, $b$ ) identifier des façons de faire singulières et en même temps reconnaître du typique et du commun à travers les différentes expériences restituées, c) de repérer, à certains traits qui commencent à s'amorcer dans la situation, qu'il est encore possible de la «redresser», de la modifier ou au contraire que celle-ci est trop dégradée et qu'il faut se tourner vers une autre configuration et d) s'approprier ce qui est perçu par d'autres utilisateurs comme une source de progrès pertinente et accessible pour eux-mêmes également compte tenu de leurs dispositions à agir du moment. 
l'acceptation de suspendre momentanément la co-enquête sur l'activité pour mieux y revenir, la prise en compte des attentes professionnelles des acteurs dans la situation constituent des moyens d'agir qui contribuent à l'enrichissement et à la pérennisation de la situation sur des temporalités assez longues (Durand, Hauw, Leblanc, Saury, \& Sève, 2005). Dans ces environnements de vidéo formation, les pistes à exploiter par les accompagnateurs consistent à susciter, à faciliter l'articulation entre mémoire, actualité et anticipation-imagination, en donnant à la fois la possibilité à l'acteur de se souvenir de conduites passées qui retrouvent un «certain coefficient de présence » dans la situation présente et aussi la possibilité de prévoir la conduite juste à venir. Nous postulons que la conscientisation d'anticipations représentées sous forme d'images et des conséquences tirées des expériences passées en lien avec la situation seront des ressources mobilisables en cours d'action, car cela constitue «par avance une expérience» (Simondon, 2008, p. 277). L'accompagnement des formateurs dans ce type de situation d'alloconfrontation vidéo est donc à penser avec plusieurs visées : a) celle d'aider les formés à se voir par procuration dans l'activité de pairs ou de quasi-pairs en prenant conscience de certaines de leurs manières d'agir et des effets potentiels sur l'activité des autres acteurs, b) celle de les faire s'interroger sur leurs croyances, convictions, dispositions à agir, façons de faire non ou peu questionnées afin d'envisager de nouveaux champs de possibles, c) celle de favoriser l'imagination de nouvelles façons d'agir en s'inspirant d'images-esquisses élaborées à partir des ressources vidéo issues de l'environnement de formation, et d) celles d'accompagner les formés dans leurs observations pour que leur analyse réflexive puisse dépasser l'émergence d'affects et s'enrichir d'autres repères favorisant la «renormalisation ${ }^{20}{ }$ " de leur conception du métier (Saujat, 2010).

\section{BIBLIOGRAPHIE}

Amalberti, R., de Montmollin, M., \& Theureau, J. (1991). Modèles en analyse du travail. Bruxelles: Margada.

Arnoud, J., \& Falzon, P. (2013). La co-analyse constructive des pratiques. In P. Falzon (Ed.), Ergonomie constructive (pp. 223-236). Paris: PUF.

Barbier, J.-M. \& Durand, M. (2003). L'activité : un objet intégrateur pour les sciences sociales? Recherche et Formation, 42, 99-117.

Bertone, S. (2011). La force des règles dans l'apprentissage du métier d'enseignant en formation par alternance. Note de synthèse pour l'Habilitation à Diriger des Recherches (non publié). Université de La Réunion.

Brouwer, C. N. (2011). Imaging Teacher Learning. A Literature Review on the Use of Digital Video for Preservice Teacher Education and Professional Development. Paper presented at the Annual Meeting of the American Eudational Research Association, New Orleans. Nijmegen: ILS Graduate School of Education, Radboud University Nijmegen.

Brunvand, S., \& Fishman, B. (2006). Investigating the impact of the availability of scaffolds on preservice teacher noticing and learning from video. Journal of Educational Technology Systems, 35(3), 151-174.

Caens-Martin, S., Specogna, A., Delépine, L., \& Girerd, S. (2004). Un simulateur pour répondre aux besoins de formation sur la taille de la vigne. Sciences et Technologies de l'Information et de la Communication pour l'Éducation et la Formation, 11. En ligne, http://sticef.univlemans.fr/num/vol2004/delepine-02/sticef_2004_delepine_02.pdf.

Calandra, B., \& Puvirajah, A. (2011). A framework for facilitating transformation in novice teachers using digital video. Educational Technology. 51(2), 33-36.

Château, J.-Y. (2008). Le vocabulaire de Simondon. Paris: Ellipse.

20 Les processus de «renormalisations » correspondent à une réappropriation par les acteurs des critères normatifs du travail pour en faire des instruments de leur propre activité. Cette reprise d'initiative qui les amène à convertir «des défenses passives en ripostes actives », passe nécessairement par des médiations collectives (Saujat, 2010, p. 90). 
Cifali Bega, M. (2009). Un sujet et son engagement dans la formation : approche clinique. In J.M. Barbier, E. Bourgeois, G. Chapelle, \& J.C. Ruano-Borbalan (Eds.), Encyclopédie de la formation (pp. 71-82). Paris: PUF.

Citton, Y. (2012). Gestes d'humanités. Paris: Armand Collin.

Clot, Y. (1999). La fonction psychologique du travail. Paris: PUF.

Daniellou, F. (2007). Des fonctions de la simulation des situations de travail en ergonomie. Activités, $4(2), 77-83$.

Durand, M. (1996). L'enseignement en milieu scolaire. Paris: PUF.

Durand, M. (2008). Un programme de recherche technologique en formation des adultes : une approche enactive de l'activité humaine et l'accompagnement de son apprentissage/développement. Éducation \& Didactique, 3(2), 97-121.

Durand, M. (2009). La conception d'environnement de formation sous le postulat de l'enaction. In M. Durand, \& L. Filliettaz (Eds.). Travail et formation des adultes (pp. 191-225). Paris: PUF.

Durand, M., Hauw, D., Leblanc, S., Saury, J., \& Sève, C. (2005). Analyse de pratiques et entraînement en sport de haut-niveau. Éducation permanente, 161, 54-68.

Durand, M., Ria, L., \& Veyrunes, P. (2010). Analyse du travail et technologie de formation : signification et organisation de l'activité des enseignants. In F. Saussez, F. Yvon, \& F. Loyola (Eds.), Analyser l'activité enseignante : des outils méthodologiques et théoriques pour l'intervention et la formation (pp. 17-39). Québec: Presses de 1'Université de Laval.Education Permanente, 2005

Durand, M., de Saint Georges, I., \& Meuwly-Bonte, M. (2006). Le curriculum en formation des adultes : Argumentation pour une approche « orientée-activité ». Raisons Educatives, 10, 185-202.

Étienne, R., Altet M., Lessard C., Paquay L., \& Perrenoud, P. (2009). Former les enseignants à l'université. Bruxelles: De Boeck.

Étienne, R., \& Bucheton D. (2009). Des gestes professionnels à l'agir des enseignants, un fil d'Ariane pour tisser la formation des enseignants et de leurs formateurs. In D. Bucheton (Ed..), L'agir enseignant : des gestes professionnels ajustés (pp. 231-241). Toulouse: Octarès.

Faïta, D. (2007). L'image animée comme artéfact dans le cadre méthodologique d'une analyse clinique de l'activité. Activités, 4(2), 3-15.

Faïta, D., \& Saujat, F. (2010). Développer l'activité des enseignants pour comprendre et transformer leur travail : un cadre théorique et méthodologique. In F. Yvon, \& F. Saussez (Eds.), Des outils théoriques et méthodologiques pour l'intervention et la formation (pp. 41-69). Laval: Presses Universitaires de Laval.

Falzon, P. (2013). Pour une ergonomie constructive. In P. Falzon (Ed.), Ergonomie constructive (pp. 1-15). Paris: PUF.

Félix C., \& Saujat F. (2008). L'aide au travail personnel des élèves entre déficit de prescriptions et « savoirs méthodologiques » : un double regard didactique et ergonomique. Les Dossiers des Sciences de l'Education, 20, 123-136.

Flandin, S. (2014). Trois approches contrastées de la vidéoformation dans le paysage francophone. Tensions épistémologiques et effets en formation. Conférence de consensus de la Chaire UNESCO «Former les enseignants au XXIe siècle », La vidéoformation dans tous ses états : quelles options théoriques? Quels scénarios? Pour quels effets? Lyon, France, 23 janvier. http://www.enslyon.fr/chaire-unesco-formation/manifestations-scientifiques/video-formation/conference-lavideoformation-dans-tous-ses-etats-1/videos

Flandin, S., \& Ria, L. (2013). Making dissatisfaction emerge about activity:Videotraining to foster teachers'professional development.Communication presented at the Néopass@ction study visit: develpment, design and study of video-training devices. IFE - ENS de Lyon, France, 26-28 mars.

Gaudin, C. (2014). Vidéoformation au plan international : quelles nouvelles voies ? Quelles recommandations, quelles zones d'ombre et perspectives ? Conférence de consensus de la Chaire UNESCO «Former les enseignants au XXIe siècle», La vidéoformation dans tous ses états : quelles options théoriques? Quels scénarios? Pour quels effets? Lyon, France, 23 janvier. http://www .ens-lyon.fr/chaire-unesco-formation/manifestations-scientifiques/videoformation/conference-la-videoformation-dans-tous-ses-etats-1/videos 
Gaudin, C., \& Chaliès, S. (2012). L'utilisation de la vidéo dans la formation professionnelle des enseignants novices : revue de littérature et zones potentielles d'étude. Revue Française de Pédagogie, 178, 115-130.

Henri, F., Compte, C., \& Charlier, B. (2007). La scénarisation pédagogique dans tous ses débats... Revue internationale des technologies en pédagogie universitaire, 4(2), 14-24.

Hotte, R., Godinet, H., \& Pernin, J.-P. (2007). Scénariser l'apprentissage, une activité de modélisation. Revue internationale des technologies en pédagogie universitaire, 4(2), 7-13.

Jullien, F. (2009). Les transformations silencieuses. Paris: Grasset.

Lahire, B. (1998). L’homme pluriel. Les ressorts de l'action. Paris: Éditions Nathan.

Lave, J. (1988). Cognition in practice: Mind, mathematics and culture in everyday life. Cambridge: Cambridge University Press.

Lave, J., \& Wenger, E. (1991). Situated learning: legitimate peripheral participation. Cambridge, UK: Cambridge University Press.

Leblanc, S. (2007). Concepts et méthodes pour valoriser l'activité professionnelle au sein de la formation initiale et continue des enseignants. Revue des Hautes Écoles pédagogiques et Institutions assimilées de Suisse Romande et du Tessin, 6, 11-33.

Leblanc, S. (2012). Conception d'environnements vidéo numériques de formation. Développement d'un programme de recherche technologique centré sur l'activité dans le domaine de l'éducation. Note de synthèse pour l'habilitation à diriger les recherches non publiée. Université Montpellier 3, Montpellier.

Leblanc, S. (2014a). Scénarios de vidéo formation au sein d'un processus progressif de professionnalisation. Conférence de consensus de la Chaire UNESCO « Former les enseignants au XXIe siècle », La vidéoformation dans tous ses états : quelles options théoriques? Quels scénarios? Pour quels effets? Lyon, France, 23 janvier. http://www.ens-lyon.fr/chaire-unescoformation/manifestations-scientifiques/video-formation/conference-la-videoformation-dans-tousses-etats-1/videos

Leblanc, S. (2014b). Observer ses collègues ou soi-même. Cahiers pédagogiques, 511, 23-25.

Leblanc, S. (sous presse). Des dispositions concurrentes pour mener un entretien post-leçon : Étude des effets d'un contexte d'entretien « innovant». In I. Plazaola Giger, \& A. Muller (Eds.), Dispositions, Travail et Formation. Toulouse: Octarès.

Leblanc, S., \& Ria, L. (2014). Designing the Néopass@ction Platform Based on Modeling of Beginning Teachers' Activity. Design and Technology Education: An International Journal, 19(2).

Leblanc, S., Ria, L., Dieumegard, G., Serres, G., \& Durand, M. (2008). Concevoir des dispositifs de formation professionnelle des enseignants à partir de l'analyse de l'activité dans une approche enactive. Activités, 5(1), 58-78.

Leblanc, S., Ria, L., \& Veyrunes, P. (2012). Vidéo et analyse in situ des situations d'enseignement et de formation dans le programme du cours d'action. In L. Veillard, \& A. Tiberghien (Eds.), Instrumentation de la recherche en Éducation. Le cas du développement d'une base de vidéos de situation d'enseignement et d'apprentissage ViSA (pp. 63-94). Paris: Maison des Sciences de l'Homme. http://books.openedition.org/editionsmsh/1930

Leblanc, S., \& Sève, C. (2012). Vidéo formation et construction de l'expérience professionnelle. Recherche et Formation, 70, 47-60.

Leblanc, S., \& Veyrunes, P. (2012). Vidéoscopie et modélisation de l'activité enseignante. Recherche et Formation, 68, 139-152.

Leplat, J. (1997). Regards sur l'activité en situation de travail. Contribution à la psychologie ergonomique. Paris: PUF.

Leplat, J., \& Hoc, J.-M. (1981). Subsequent verbalization in the study of cognitive processes. Ergonomics, 24(10), 743-755.

Linard, M. (2002). Conception de dispositifs et changement de paradigme en formation. Éducation Permanente, 152, 143-155.

Lussi-Borer, V., \& Muller, A. (sous presse). Quel apport/usage du « voir » pour le «faire » en formation des enseignants du secondaire. In L. Paquay, M. Altet, J. Desjardins, R. Etienne, \& P. Perrenoud (Eds.). Prendre en compte le travail en formation des enseignants. Bruxelles: De 
Boeck.

Mayen, P. (1999). Des situations potentielles de développement. Éducation Permanente, 139, 65-86.

Mayen, P. (2009). Expérience et formation des adultes : place et fonction de l'expérience en formation des adultes. In J.M. Barbier, E. Bourgeois, G. Chapelle, \& J.C., Ruano-Borbalan (Eds.), Encyclopédie de la formation (pp. 763-780). Paris: PUF.

Méard, J. (2004). L'analyse de pratique au quotidien. Éducation permanente, 161(2), 45-53.

Merleau-Ponty, M. (1942). La structure du comportement. Paris: P.U.F.

Mollo, V., \& Falzon, P. (2004). Auto- and allo-confrontation as tools for reflective activities. Applied Ergonomics, 35(6), 531-540.

Moussay, S. (2013). Conception d'un dispositif de vidéo-formation centré sur le travail réel et professionnalisation au métier d'enseignant en STAPS. eJRIEPS, 29, 4-26.

Ouellet, S. (2013). Contribution de l'ergonomie à la conception d'un outil de formation. Activités, 10(2), 3-19, http://www .activites.org/v10n2/v10n2.pdf

Pastré, P. (1995). Problèmes didactiques posés par les simulations. Performances Humaines et Techniques, 75/76, 44-53.

Pastré, P. (2005). Apprendre par la simulation. De l'analyse du travail aux apprentissages professionnels. Toulouse: Octarès.

Perrenoud, P. (2001). Développer la pratique réflexive dans le métier d'enseignant. Paris: ESF.

Prusak, K., Dye, B., Graham, C., \& Graser, S. (2010). Reliability of pre-service physical education teachers'coding of teaching videos using studiocode analysis software. Journal of Technology and Teacher Education, 18(1), 131-159. Chesapeake, VA: Association for the Advancement of Computing in Education.

Quéré, L. (1993). Langage de l'action et questionnement sociologique. In P. Ladrière, P. Pharo, \& L. Quéré (Eds.), La théorie de l'action. Le sujet pratique en débat (pp. 53-83). Paris: CNRS Éditions.

Ria, L. (2009). De l'analyse de l'activité des enseignants débutants en milieu difficile à la conception de dispositifs de formation. In M. Durand, \& L. Filliettaz (Eds.), Travail et formation des adultes (pp. 217-243). Paris: PUF.

Ria, L. (Ed.) (2010). Plateforme de formation en ligne « Néopass@ction » de l'Institut Français de l'Éducation de l'ENS de Lyon. http://neo.ens-lyon.fr/neo

Ria, L., \& Leblanc, S. (2011). Conception de la plateforme de formation Néopass@ @tion à partir d'un observatoire de l'activité des enseignants débutants : enjeux et processus. Activités, 8(2), 112-134. http://www .activites.org/v8n2/v8n2.pdf

Ria, L., \& Leblanc, S. (2012). Professionnalisation assistée par vidéo : Les effets d'une navigation sur Néopass@ction.Recherches et Éducations, 7, 99-114.

Rix-Lièvre, G. (2010). Différents modes de confrontation à des traces de sa propre activité. Vers une confrontation à une perspective subjective située. Revue d'anthropologie des connaissances, 4(2), 358-379.

Rogalski, J. (2003). Y a-t-il un pilote dans la classe ? Une analyse de l'activité de l'enseignant comme gestion d'un environnement dynamique ouvert. Recherches en Didactique des Mathématiques, 23(3), 343-388.

Rosaen C., Lundeberg M., Cooper M., Fritzen A., \& Terpstra M. (2008). Noticing noticing: How does investigation of video records change how teachers reflect on their experiences? Journal of Teacher Education, 59(4), 347-360.

Salembier, P., Theureau, J., Zouinar, M., \& Vermersch, P. (2001). Action/cognition située et assistance à la coopération. Communication présentée aux $12^{\text {ème }}$ Journées Francophones d'Ingénierie des Connaissances (IC'2001), Grenoble, France, Juin.

Salembier, P., \& Zouinar, M. (2004). Intelligibilité mutuelle et contexte partagé : Inspirations théoriques et réductions technologiques. Activités, 1(2), 64-85. http://www .activites.org/v1n2/salembier.pdf

Santagata R. (2009). Designing video-based professional development for mathematics teachers in low-performing schools. Journal of Teacher Education, 60, 1, 38-51.

Saujat, F. (2010). Travail, formation et développement des professionnels de l'éducation : voies de 
recherche en sciences de l'éducation. Note de synthèse pour l'Habilitation à Diriger des Recherches (non publiée). Université de Provence.

Saury, J. (2008). La coopération dans les situations d'intervention, de performance et d'apprentissage en contexte sportif. Contribution au développement d'un programme de recherche en ergonomie cognitive des situations sportives en STAPS. Note de synthèse pour l'habilitation à diriger des recherches non publiée. Université de Nantes, Nantes.

Schön, D.A., (1994). Le praticien réflexif. À la recherche du savoir caché dans l'agir professionnel. Montréal: Les Éditions Logiques.

Sherin M. (2004). New perspectives on the role of video in teacher education. In J. Brophy (dir.), Using video in teacher education (pp. 1-28). Oxford: Elsevier.

Seidel, T., Blomberg, G., \& Renkl, A. (2013). Instructional strategies for using video in teacher education. Teaching and Teacher Education, 34, 56-65.

Serres, G. (2006). Analyse de la construction de l'expérience professionnelle au gré des diverses situations de formation initiale des enseignants du second degré. Thèse de doctorat non publiée. Université de Blaise Pascal, Clermont-Ferrand.

Sève, C., \& Saury, J. (2010). Un programme de recherche en STAPS fondé sur la théorie du cours d'action. Ejrieps, 19, 5-25.

Simondon, G. (1989). L'individuation psychique et collective. Paris: Aubier.

Simondon, G. (2005). L'individuation à la lumière des notions de forme et d'information. Grenoble: Million.

Simondon, G. (2008). Imagination et invention. Lonrai : La transparence.

Six-Touchard, B., \& Falzon, P. (2013). L'auto-analyse du travail : une ressource pour le développement des compétences. In P. Falzon (Ed.), Ergonomie constructive (pp. 223-23. Paris: PUF.

Stiegler, B. (2010). Philosopher par accident. Paris: Galilée.

Suchman, L. A. (1987). Plans and Situated Actions. Cambridge: Cambridge University Press.

Theureau, J. (2000). Anthropologie cognitive \& analyse des compétences. In J.-M. Barbier (Ed.). L'analyse de la singularité de l'action. pp. 171-211. Paris : PUF.

Theureau, J. (2004). Cours d'action: méthode élémentaire. Toulouse: Octarès.

Theureau, J. (2006). Cours d'action: méthode développée. Toulouse: Octarès.

Theureau, J. (2010). Les entretiens d'autoconfrontation et de remise en situation par les traces matérielles et le programme de recherche «cours d'action ». Revue d'anthropologie des connaissances, 4(2), 287-322.

Theureau, J. (2012). Nouveaux essais de méthode réfléchie 1 : Reconsidérer l'épistémologie générale à partir de la considération de l'activité de recherche? Révision de la section 2. pp. 451-460 du chapitre 5 de «Le cours d'action : Méthode réfléchie » (2009).

Theureau, J., Durand, M., Leblanc, S., Saury, J., \& Sève, C. (2004). Étude sémiologique des activités humaines, recherche et conception en formation et éducation. Texte non publié.

Theureau, J., \& Jeffroy, F. (1994). Ergonomie des situations informatisées : la conception centrée sur le cours d'action des utilisateurs. Toulouse: Octarès.

van Es E., \& Sherin M. (2008). Mathematics teachers" "learning to notice" in the context of a video club. Teaching and Teacher Education, 24(2), 244-276.

Varela, F.J. (1989). Connaître les sciences cognitives. Paris: Seuil.

Varela, F.J., Thompson, E., \& Rosch, E. (1993). L'inscription corporelle de l'esprit. Paris: Seuil.

Veyrunes, P. (2011). Formats pédagogiques et configuration de l'activité collective à l'école primaire. Note de synthèse pour l'Habilitation à Diriger des Recherches (non publiée). Université de Toulouse 2, Le Mirail.

Vermersch, P. (2006). L'entretien d'explicitation. Paris: ESF.

Winograd, T., \& Florès, F. (1989). L'intelligence artificielle en question. Paris: PUF. 


\section{RESUME}

Ce texte présente les principaux résultats d'une étude longitudinale, qui révèle comment une enseignante débutante peut acquérir de l'expérience professionnelle par procuration et anticipation via une plateforme de vidéo formation en ligne, centrée sur l'analyse du travail réel des enseignants. Pour pister les transformations de l'activité potentiellement induites par plusieurs navigations libres en présence et avec l'aide d'un chercheur-interlocuteur, le «cours de vie » d'une enseignante, débutante dans le métier, relatif à l'utilisation de cette plateforme (Theureau, 2004) a été reconstruit sur la base d'un protocole à trois volets articulant les ressources vidéo consultées, les verbalisations simultanées durant les sessions et un retour d'expérience réalisé un mois plus tard. Les résultats présentent a) l'analyse locale de l'élaboration d'une autotransformation projetée en lien avec son contexte professionnel, b) une synthèse de la reconstruction des principales tensions professionnelles vécues sur les 6 premiers mois de classe et révélées lors des navigations sur la plateforme et, c) les effets produits sur son activité en classe à partir du suivi de certaines de ces auto-prescriptions. À partir des concepts d'enaction (Varela, 1989) et d'individuation (Simondon, 2005), nous discutons ces résultats et proposons une certaine vision de l'apprentissagedéveloppement et des processus d'intégration dans un collectif.

\section{MOTS-CLES}

formation des enseignants, analyse de l'activité, cours de vie, expérience mimétique, alloconfrontation

\section{REFERENCEMENT}

Leblanc, S. (2014). Vidéo formation et transformations de l'activité professionnelle. Activités, 11(2), 143-171. htpp://www .activites.org/v11n2/v11n2.pdf

Article soumis le 7 janvier 2014, accepté pour publication le 20 avril 2014 
Annexe 1 : Synoptique des vidéos consultées sur les trois sessions de navigation Appendix 1: Overview of videos consulted on the navigation of three Neopass@ction sessions

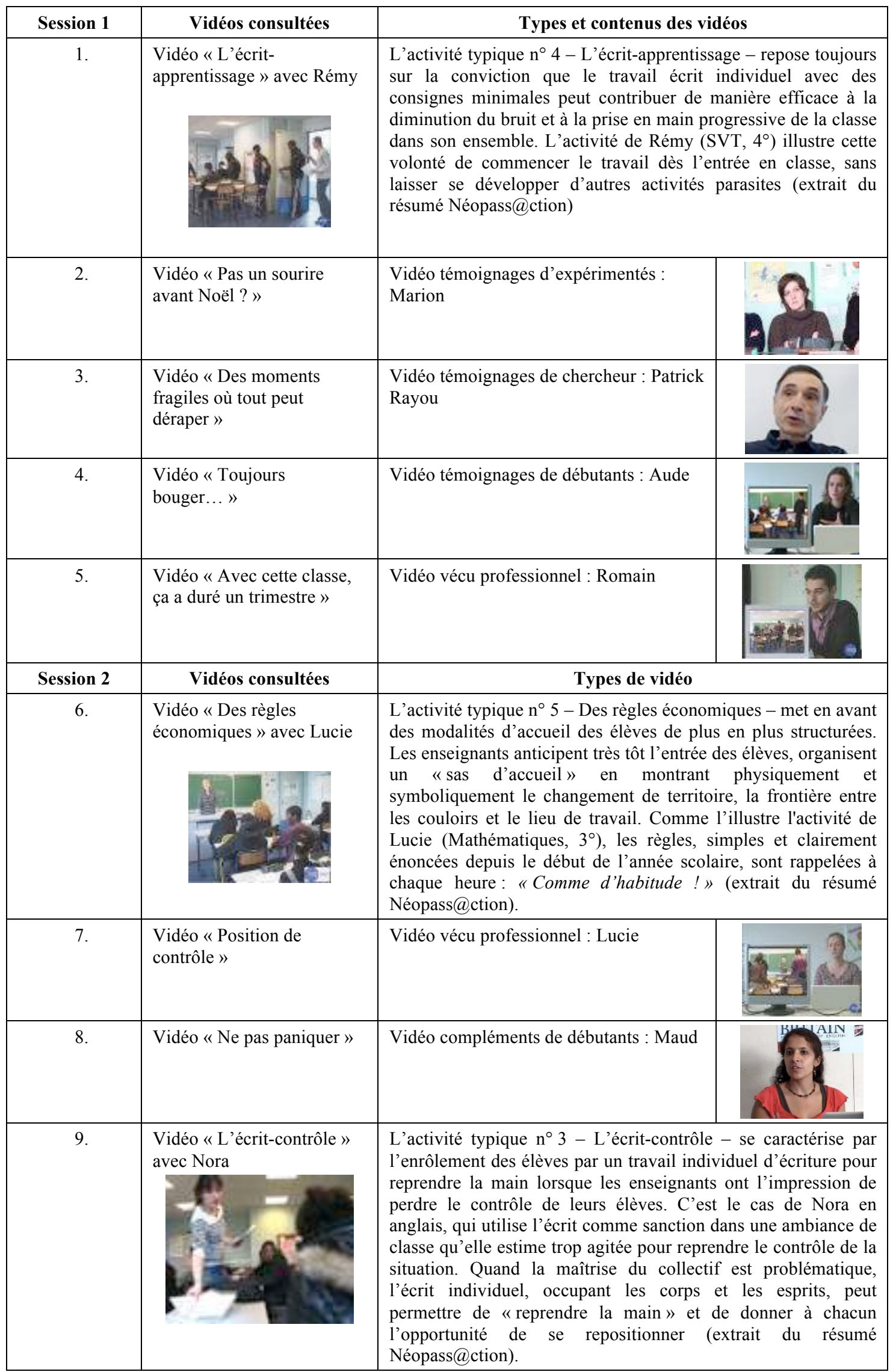




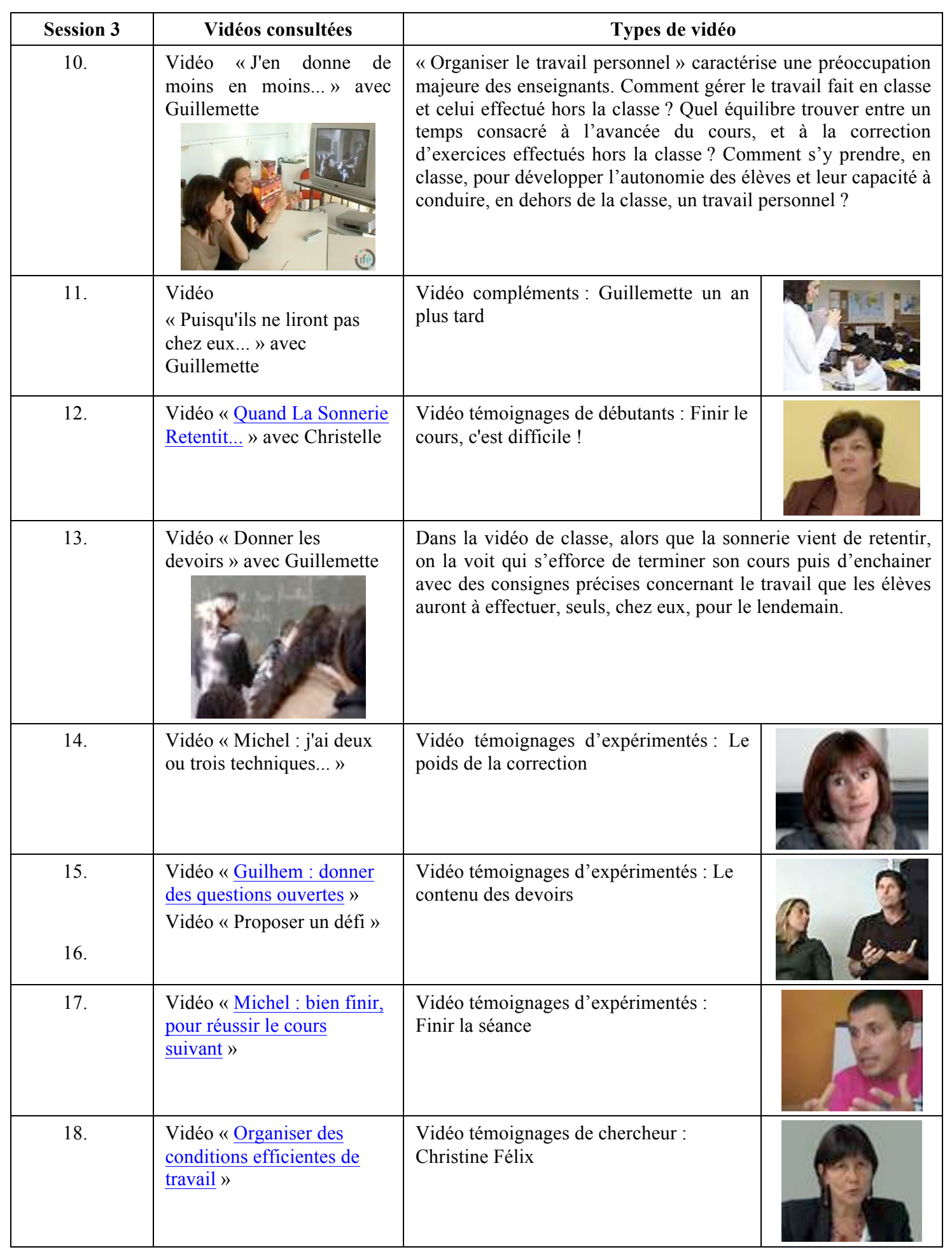


Annexe 2 : Extrait du récit réduit et identification de la séquence discontinue 1 (en gris) «Contrôler la mise en position scolaire de tous les élèves lors du début de cours à travers des gestes adaptés de l'enseignant»

Appendix 2: Concatenation of elementary units of meaning and identification of discontinuous sequence 1 (gray) "Monitoring the academic positioning of all students at the beginning of the course through gestures adapted from the teacher"

\begin{tabular}{|c|c|}
\hline \multicolumn{2}{|r|}{ Récit réduit de la navigation commentée de Coralie au chercheur : enchaînement des unités d'expérience } \\
\hline 1. & $\begin{array}{l}\text { Avant d'avoir commencé la navigation dans la plateforme, précise au chercheur qu'elle recherche des gestes et une manière de faire } \\
\text { pour mettre tous les élèves au travail lorsqu'ils ne sont pas enclins à le faire }\end{array}$ \\
\hline 2. & $\begin{array}{l}\text { Lit le résumé correspondant à la vignette «écrit-apprentissage » dans le thème } 1 \text { « début de cours et mise au travail des élèves » pour } \\
\text { évaluer si cela correspond à ses attentes }\end{array}$ \\
\hline 3. & Lance la vidéo « écrit-apprentissage » en se disant que c’est un moyen de les mettre au travail d'entrée \\
\hline 4. & Estime à l'issue du visionnement de la vidéo que ça marche bien pour les faire se calmer et se mettre dans des conditions de travail \\
\hline 5. & S'interroge sur l'absence d'explication collective de la consigne de travail et sur le caractère noté ou non de l'exercice proposé \\
\hline 6. & Évoque un contrôle sur table qu'elle a donné en tout début de cours que lui rappelle cette entrée en classe \\
\hline 7. & S'interroge sur la possibilité de faire cela systématiquement ou bien de ne le réserver qu'à des moments perçus comme difficiles \\
\hline 8. & Imagine une manière d'agir différente de celle de l'enseignant visant à proscrire les échanges entre les élèves \\
\hline 9. & $\begin{array}{l}\text { Interpellée par le titre d'une vignette vidéo «pas un sourire avant Noël », fait le lien avec des propos sur le même objet qu'on lui avait } \\
\text { tenu en stage et qui l'avaient choqué }\end{array}$ \\
\hline 10. & Lance la vidéo pour confronter son point de vue « on peut se permettre de sourire tout en gardant une certaine autorité » à ce qui est dit \\
\hline 11. & Compare les propos de l'enseignante dans la vidéo avec le discours qu'on lui avait tenu en stage \\
\hline 12. & Enonce les conseils qu'on lui avait donnés en stage : «être clair », « être ferme » \\
\hline 13. & $\begin{array}{l}\text { Évoque en réponse à la question du chercheur ce sur quoi elle est particulièrement exigeante en début d'année : «le travail dans le } \\
\text { silence » }\end{array}$ \\
\hline 14. & Évoque à la suite qu'elle est un peu en difficulté pour contraindre les élèves à lever la main \\
\hline 15. & $\begin{array}{l}\text { En écho aux propos de l'enseignant «si tu n’y arrives pas, il faut changer de métier », se rappelle que son principal lui a fait une } \\
\text { remarque du même ordre en précisant que les élèves de son collège étaient calmes }\end{array}$ \\
\hline 16. & Évoque les propos rassurant de sa tutrice qui nuançaient voire s'opposaient à ceux du principal \\
\hline 17. & Reste convaincue que l'on peut sourire avant Noël \\
\hline 18. & Choisit de regarder la vidéo d'un chercheur intitulé « des moments fragiles où tout peut déraper » \\
\hline 19. & S'interroge sur sa manière de donner les devoirs en fin de cours qui ne fonctionne pas toujours \\
\hline 20. & Évoque d'autres alternatives possibles \\
\hline 21. & Se sent un peu déstabilisé par ces interrogations \\
\hline 22. & Ne voit pas de quelle façon par contre il s'agit d'un moment où ça peut déraper \\
\hline 23. & Expose son rituel de sortie de classe qui est basé sur le silence \\
\hline 24. & Rejoue des interactions élèves-enseignant suite à des revendications d'élèves « Madame, ça a sonné » dans une fin de cours \\
\hline 25. & $\begin{array}{l}\text { En écho à l'évocation des sorties du professeur d'EPS par le chercheur comme exemple de moment à risques, se souvient qu'elle doit } \\
\text { accompagner une sortie d'accrobranche la semaine suivante }\end{array}$ \\
\hline 26. & Appréhendant cette sortie, se demande si elle peut trouver des pistes pour gérer une classe en dehors d'une salle de classe \\
\hline 27. & Demande explicitement au chercheur s'il peut la guider pour trouver une vidéo en lien avec son attente \\
\hline 28. & $\begin{array}{l}\text { Consulte la vidéo conseillée par le chercheur d'une professeure d'EPS qui commente la première vidéo de classe de Romain } \\
\text { (enseignant en difficulté pour gérer le début de son cours) }\end{array}$ \\
\hline 29. & Exprime son accord avec les propos de la collègue sur les conditions à respecter pour que la position « les bras croisés » soit efficace \\
\hline 30. & Rejoue ce qu'elle fait dans sa propre classe en décrivant précisément certaines attitudes d'élèves \\
\hline 31. & $\begin{array}{l}\text { Imagine une alternative à tester dans sa classe qui intègre des pistes issues des propos de la collègue d'EPS jugées pertinentes pour elle } \\
\text { pour que les élèves se mettent plus rapidement au travail }\end{array}$ \\
\hline 32. & Est intéressée pour voir comment Romain analyse son activité et à quoi il pensait durant cette attente \\
\hline 33. & Manifeste son désaccord avec le propos de Romain « c’est eux qui décident » (les élèves) \\
\hline 34. & Remet en cause le poids de l'argument développé par Romain relatif au brevet pour les responsabiliser \\
\hline 35. & Manifeste son accord avec Romain sur sa préférence à se mettre en position de retrait plutôt que de crier \\
\hline 36. & Interpellée par la position de Romain adossé au tableau les bras croisés (image fixe), arrête sa consultation \\
\hline 37. & Se rappelle qu'une formatrice lui avait fait cette même remarque \\
\hline 38. & Prend conscience de l'image que cela peut renvoyer aux élèves et de ses effets \\
\hline 39. & $\ldots$ \\
\hline
\end{tabular}

